\title{
NUMERICAL ANALYSIS OF A STABILIZED FINITE ELEMENT APPROXIMATION FOR THE THREE-FIELD LINEARIZED VISCOELASTIC FLUID PROBLEM USING ARBITRARY INTERPOLATIONS
}

\author{
E. CASTILlO ${ }^{1}$ AND R. Codina ${ }^{2}$
}

\begin{abstract}
In this paper we present the numerical analysis of a three-field stabilized finite element formulation recently proposed to approximate viscoelastic flows. The three-field viscoelastic fluid flow problem may suffer from two types of numerical instabilities: on the one hand we have the two inf-sup conditions related to the mixed nature problem and, on the other, the convective nature of the momentum and constitutive equations may produce global and local oscillations in the numerical approximation. Both can be overcome by resorting from the standard Galerkin method to a stabilized formulation. The one presented here is based on the subgrid scale concept, in which unresolvable scales of the continuous solution are approximately accounted for. In particular, the approach developed herein is based on the decomposition into their finite element component and a subscale, which is approximated properly to yield a stable formulation. The analyzed problem corresponds to a linearized version of the Navier-Stokes/Oldroyd-B case where the advection velocity of the momentum equation and the non-linear terms in the constitutive equation are treated using a fixed point strategy for the velocity and the velocity gradient. The proposed method permits the resolution of the problem using arbitrary interpolations for all the unknowns. We describe some important ingredients related to the design of the formulation and present the results of its numerical analysis. It is shown that the formulation is stable and optimally convergent for small Weissenberg numbers, independently of the interpolation used.
\end{abstract}

1991 Mathematics Subject Classification. 65N12, 76A10, 76M10.

\section{INTRODUCTION}

The numerical approximation of viscoelastic fluid flows is a current line of research due to the wide range of industrial applications where these materials are found [15]. The mathematical structure of the equations

Keywords and phrases: Stabilized finite element methods, viscoelastic fluids, Oldroyd-B, inf-sup conditions.

${ }^{1}$ Universitat Politècnica de Catalunya, Jordi Girona 1-3, Edifici C1, 08034, Barcelona, Spain

Universidad de Santiago de Chile (USACH), Departamento de Ingeniería Mecánica, Av. Bdo. O’Higgins 3363, Santiago, Chile

${ }^{2}$ Universitat Politècnica de Catalunya, Jordi Girona 1-3, Edifici C1, 08034, Barcelona, Spain CIMNE - Centre Internacional de Metodes Numerics en Enginyeria, Gran Capità S/N 08034 Barcelona, Spain

(C) EDP Sciences, SMAI 1999 
that define the problem presents different types of numerical instabilities and difficulties in which intensive research has been done in recent years (see for example $[1,25,37]$ for a review about this topic).

When using finite elements, the numerical approximation of the flow of viscoelastic fluids presents basically two types of instabilities. The first is associated with the two compatibility or inf-sup conditions that restrict the possible choices of interpolation spaces. The second is related to the convective terms in both the momentum and the hyperbolic constitutive equation, in both cases nonlinear.

Referring to the compatibility conditions, many authors have studied the problem, considering first simplified model problems such as the three-field Stokes problem $[9,18,35]$ or the three-field Navier-Stokes problem [3,12], which have the same interpolation requirements as the viscoelastic case. For these simplified problems the classical compatibility condition between velocity and pressure [10] is not sufficient, and an additional condition that relates the interpolation of the velocity with that of the elastic stress must be added. The convective terms have been treated using the extension of classical methods used for the Navier-Stokes problem, such as the SUPG method [34], the Galerkin/Least-Squares (GLS) method [23] or approaches based on the Variational Multiscale (VMS) concept (see [13,31], among others).

Concerning the mathematical analysis, the existence of a slow steady viscoelastic flow solution has been proved by Renardy in [36]. For the time-dependent case, existence of solutions locally in time, and for small data globally in time, have been proved by Guillopé and Saut [26]. These analysis have been performed in Hilbert spaces. The extension to Banach spaces and a complete review of uniqueness, regularity, wellposedness and stability results can be found in the work of Fernández-Cara et al. [25]. The existence of global weak solutions for general initial conditions using a co-rotational Oldroyd model has been proved by Lions and Masmoudi in [33].

In the context of the finite element approximation, for the steady state case one of the first works where the existence of approximate solutions and error analysis were presented is that of Baranger and Sandri in [2]. The authors used a discontinuous interpolation (Lesaint-Raviart method) to treat the viscoelastic stresses. Later, Sandri in [40] showed by using a fixed point method that the discrete approximate problem using a $P 1$ (continuous)- $P 2$ (continuous)- $P 1$ (continuous) interpolation for stress, velocity and pressure, respectively, and the SUPG method to treat the convective term in the constitutive equation, has an unique solution for which error bounds were given, supposing that the continuous problem admits sufficiently smooth and small solutions. Picasso and Rappaz [35] analyzed a stationary non-linear Stokes problem (Stokes/OldroydB model without convective term), and they proved a priori and a posteriori error estimates for the finite element approximation for small Weissenberg numbers using a GLS method and an Elastic Viscous Split Stress (EVSS) scheme. The extension to the time-dependent case was treated in [8] for the same non-linear Stokes problem, proving global existence in time in Banach spaces provided the data are small enough. For a Stokes/Oldroyd-B linearized problem, Bonito and Burman presented in [7] optimal a priori error estimates using the Interior-Penalty method. In this work the authors showed that adding some type of artificial viscosity in the constitutive equation, the problem can be solved for a large range of Weissenberg numbers. A similar problem was studied by Ervin et al. in [21] for the steady state case, but using the Johnson-Segalman linearized constitutive model, proving existence and uniqueness of the continuous problem and of a finite element approximation under small data assumption. Ervin and Miles in [22] analyzed the Oldroyd-B timedependent case both in the semi-discrete and in the fully discrete cases using the SUPG method, proving 
existence and deriving a priori error estimates for the numerical approximation, assuming a Taylor-Hood pair approximation for the velocity and pressure and a continuous approximation for the viscoelastic stresses.

The stabilized finite element formulation analyzed in this work has its roots in the VMS framework introduced by Hughes et al. [28] for the scalar convection-diffusion-reaction problem, and extended later to the vectorial Stokes problem in [16], where the space of the sub-grid scales is taken orthogonal to the finite element space. As we shall see, this is an important ingredient in the design of the formulation analyzed herein. The starting point of a VMS approach is to split the unknowns of the problem in two scales, the finite element one and the unresolvable one, called sub-grid scale or simply subscale in what follows. The latter needs to be approximated in a simple manner in terms of the former, so as to capture its main effect and yield a stable formulation for the finite element unknown, keeping therefore the number of degrees of freedom of the Galerkin method.

One of the most common viscoelastic constitutive models used in the rheological community is the OldroydB model. When this model is approximated numerically, the most challenging problem associated to it is the High Weissenberg Number Problem (HWNP), a phenomenon related to the inability of the numerical algorithms to converge to a solution when the Weissenberg (or Deborah) number is high (see for example $[29,38]$ and the references therein). Some numerical tools have been proved to increase the limits of the numerical formulations to solve more elastic fluids. The log-conformation formulation presented by Fattal and Kupferman [24] is a common possibility to deal with the exponential growth of stresses when the Weissenberg number is increased. Continuation methods are another numerical tool used to increase the Weissenberg number limits that can be reached by a standard formulation, as one can see in the work of Howell [27]. For the treatment of local oscillations, discontinuity-capturing techniques have proved to produce good results in $[7,13]$.

The objective of this paper is to analyze numerically a stabilized finite element formulation presented in previous works and tested numerically with very good accuracy and robustness properties, both in stationary [13] and time dependent cases $[11,14]$. The analysis is standard and follows a classical approach to prove stability and convergence, first using a mesh-dependent or working norm, and then extending the results to natural norms, that is to say, to the norms of the spaces where the continuous problem is posed. As it is common to other analyses, the Weissenberg number needs to be small enough.

The paper is organized as follows. In the following section we present the problem to be solved and its Galerkin finite element approximation, explaining the sources of numerical instability. Then, in Section 3 we present our stabilized finite element formulation. In Section 4, we present the numerical analysis performed, and finally, conclusions and remarks are given in Section 5.

\section{Problem statement and Galerkin finite element discretization}

\subsection{Boundary value problem}

The stationary linearized viscoelastic fluid flow problem is defined by the following equations. First we have the conservation equations for momentum and mass

$$
\begin{aligned}
\rho \boldsymbol{a} \cdot \nabla \boldsymbol{u}-\nabla \cdot \boldsymbol{T}+\nabla p=\boldsymbol{f} & \text { in } \Omega \\
\nabla \cdot \boldsymbol{u}=0 & \text { in } \Omega
\end{aligned}
$$


where $\boldsymbol{a}$ is the advection velocity vector, $\Omega$ represents the computational domain of $\mathbb{R}^{d}$ occupied by the fluid, $d=2$ or 3 being the space dimensions, $\rho$ denotes the density of the fluid, $p: \Omega \longrightarrow \mathbb{R}$ the pressure, $\boldsymbol{u}: \Omega \longrightarrow \mathbb{R}^{d}$ the velocity field, $\boldsymbol{f}: \Omega \rightarrow \mathbb{R}^{d}$ the force vector and $\boldsymbol{T}: \Omega \longrightarrow \mathbb{R}^{d} \otimes \mathbb{R}^{d}$ the deviatoric extra stress tensor, which can be defined in terms of a viscous and a viscoelastic or elastic contribution as

$$
\boldsymbol{T}=2 \beta \mu \nabla^{\mathrm{s}} \boldsymbol{u}+\boldsymbol{\sigma}
$$

where, $\mu$ represents the total viscosity, $\beta \in(0,1)$ is a real parameter to define the amount of viscous or solvent viscosity $\mu_{s}=\beta \mu$ and elastic or polymeric viscosity $\mu_{p}=(1-\beta) \mu$ in the fluid, and $\nabla^{\mathrm{s}} \boldsymbol{u}$ is the symmetrical part of the gradient of $\boldsymbol{u}$, given by

$$
\nabla^{\mathrm{s}} \boldsymbol{u}:=\frac{1}{2}\left[\nabla \boldsymbol{u}+(\nabla \boldsymbol{u})^{T}\right]
$$

In (1)-(2) and in what follows we use classical tensor notation; in particular, the dot stands for the contraction of the nearest indexes of the tensors being multiplied.

We will consider that both the density $\rho$ and the total viscosity $\mu$ are constants. For viscoelastic fluids, the problem is incomplete without the definition of a constitutive equation for the elastic part of the extra stress tensor $(\boldsymbol{\sigma})$. A large variety of approaches exist to define it (see $[4,5]$ for a description). In this work, we use the classical Oldroyd-B constitutive model to describe the fluid, which is defined as

$$
\frac{1}{2 \mu} \boldsymbol{\sigma}-(1-\beta) \nabla^{\mathrm{s}} \boldsymbol{u}+\frac{\lambda}{2 \mu}\left(\boldsymbol{a} \cdot \nabla \boldsymbol{\sigma}-\boldsymbol{\sigma} \cdot \nabla \boldsymbol{a}-(\nabla \boldsymbol{a})^{T} \cdot \boldsymbol{\sigma}\right)=\mathbf{0} \text { in } \Omega
$$

where $\lambda$ is the relaxation time. This equation can be viewed as a convection-reaction equation.

Equations (1)-(4) are a mixed elliptic-hyperbolic system which additionally needs boundary conditions both in the velocity and in the stress fields to close the problem. In principle the elastic stresses can be fixed only on the inflow part of the boundary $\Gamma_{\text {in }}=\{\boldsymbol{x} \in \partial \Omega \mid(\boldsymbol{u} \cdot \boldsymbol{n})(\boldsymbol{x})<0\}$, where $\boldsymbol{n}$ is the outward unit normal vector to $\partial \Omega$. For simplicity in the exposition, we shall consider the simplest boundary condition $\boldsymbol{u}=\mathbf{0}$ on $\partial \Omega$ for the velocity.

Calling $\boldsymbol{U}=[\boldsymbol{u}, p, \boldsymbol{\sigma}], \boldsymbol{F}=[\boldsymbol{f}, 0, \mathbf{0}]$ and defining

$$
\mathcal{L}(\boldsymbol{U}):=\left(\begin{array}{c}
-2 \beta \mu \nabla \cdot \nabla^{\mathrm{s}} \boldsymbol{u}+\rho \boldsymbol{a} \cdot \nabla \boldsymbol{u}-\nabla \cdot \boldsymbol{\sigma}+\nabla p \\
\nabla \cdot \boldsymbol{u} \\
\frac{1}{2 \mu} \boldsymbol{\sigma}-(1-\beta) \nabla^{\mathrm{s}} \boldsymbol{u}+\frac{\lambda}{2 \mu}\left(\boldsymbol{a} \cdot \nabla \boldsymbol{\sigma}-\boldsymbol{\sigma} \cdot \nabla \boldsymbol{a}-(\nabla \boldsymbol{a})^{T} \cdot \boldsymbol{\sigma}\right)
\end{array}\right)
$$

we may write (1), (2) and (4) as $\mathcal{L}(\boldsymbol{U})=\boldsymbol{F}$.

This linearized problem can be viewed as a viscoelastic Oseen problem that represents a linearization of the stationary Navier-Stokes/Oldroyd-B problem, case in which $\boldsymbol{a}$ can be considered the velocity field evaluated in a previous iteration. It also appears as one of the steps of some multilevel or multi-grid methods (see for example [32] in the context of viscoelastic flows). This is why it is often used as a first step towards the analysis of the full nonlinear problem, both to obtain a priori and posteriori error estimates. With respect to the advection velocity, we will take it in $\mathcal{C}^{0}(\bar{\Omega})$, weakly divergence free and with bounded derivatives (see assumption $\mathbf{H 1}$ in the following section). 


\subsection{Variational form of the problem}

Let us introduce some notation in order to write the weak form of the problem. The space of square integrable functions in a domain $\omega$ is denoted by $L^{2}(\omega)$, and the space of functions whose distributional derivatives of order up to $m \geq 0$ (integer) belong to $L^{2}(\omega)$ by $H^{m}(\omega)$. The space $H_{0}^{1}(\omega)$ consists of functions in $H^{1}(\omega)$ vanishing on $\partial \omega$. The topological dual of $H_{0}^{1}(\Omega)$ is denoted by $H^{-1}(\Omega)$, the duality pairing by $\langle\cdot, \cdot\rangle$, and the $L^{2}$ inner product in $\Omega$ (for scalars, vectors and tensors) is denoted by $(\cdot, \cdot)$. The integral of the product of two functions in a domain $\omega$ is denoted as $\langle\cdot, \cdot\rangle_{\omega}$. The subscript $\omega$ is omitted when $\omega=\Omega$. The norm in a space $X$ is written as $\|\cdot\|_{X}$, with the subscript omitted when $X=L^{2}(\Omega)$. When $X=L^{2}(\omega)$ we denote the norm by $\|\cdot\|_{\omega}$.

Let $\boldsymbol{\Upsilon}:=\left\{\boldsymbol{\tau} \mid \boldsymbol{\tau} \in\left(L^{2}(\Omega)\right)^{d \times d}, \tau_{i j}=\tau_{j i}, \boldsymbol{a} \cdot \nabla \boldsymbol{\tau} \in\left(L^{2}(\Omega)\right)^{d \times d}\right\}, \mathcal{V}:=\left(H_{0}^{1}(\Omega)\right)^{d}$ and $\mathcal{Q}:=L^{2}(\Omega) / \mathbb{R}$, which are the spaces where we may seek the elastic stress, the velocity and the pressure, respectively. The weak form of the problem is obtained by testing (5) against an arbitrary test function $\boldsymbol{V}=[\boldsymbol{v}, q, \boldsymbol{\tau}]$ with appropriate regularity. It can be written as: find $\boldsymbol{U}=[\boldsymbol{u}, p, \boldsymbol{\sigma}] \in \mathcal{X}:=\mathcal{V} \times \mathcal{Q} \times \boldsymbol{\Upsilon}$ such that

$$
\begin{aligned}
2 \beta \mu\left(\nabla^{\mathrm{s}} \boldsymbol{u}, \nabla^{\mathrm{s}} \boldsymbol{v}\right)+\langle\rho \boldsymbol{a} \cdot \nabla \boldsymbol{u}, \boldsymbol{v}\rangle+\left(\boldsymbol{\sigma}, \nabla^{\mathrm{s}} \boldsymbol{v}\right)-(p, \nabla \cdot \boldsymbol{v}) & =\langle\boldsymbol{f}, \boldsymbol{v}\rangle \\
(q, \nabla \cdot \boldsymbol{u}) & =0 \\
\frac{1}{2 \mu}(\boldsymbol{\sigma}, \boldsymbol{\tau})-\left((1-\beta) \nabla^{\mathrm{s}} \boldsymbol{u}, \boldsymbol{\tau}\right)+\frac{\lambda}{2 \mu}\left(\boldsymbol{a} \cdot \nabla \boldsymbol{\sigma}-\boldsymbol{\sigma} \cdot \nabla \boldsymbol{a}-(\nabla \boldsymbol{a})^{T} \cdot \boldsymbol{\sigma}, \boldsymbol{\tau}\right) & =0
\end{aligned}
$$

for all $\boldsymbol{V}=[\boldsymbol{v}, q, \boldsymbol{\tau}] \in \mathcal{X}$, where it is assumed that $\boldsymbol{f}$ is such that $\langle\boldsymbol{f}, \boldsymbol{v}\rangle$ is well defined.

In a compact form, problem (6)-(8) can be written as

$$
B(\boldsymbol{U}, \boldsymbol{V})=\langle\boldsymbol{f}, \boldsymbol{v}\rangle
$$

for all $\boldsymbol{V} \in \mathcal{X}$, where

$$
\begin{aligned}
B(\boldsymbol{U}, \boldsymbol{V})= & 2 \beta \mu\left(\nabla^{\mathrm{s}} \boldsymbol{u}, \nabla^{\mathrm{s}} \boldsymbol{v}\right)+\langle\rho \boldsymbol{a} \cdot \nabla \boldsymbol{u}, \boldsymbol{v}\rangle+\left(\boldsymbol{\sigma}, \nabla^{\mathrm{s}} \boldsymbol{v}\right)-(p, \nabla \cdot \boldsymbol{v})+(q, \nabla \cdot \boldsymbol{u})+\frac{1}{2 \mu}(\boldsymbol{\sigma}, \boldsymbol{\tau}) \\
& -(1-\beta)\left(\nabla^{\mathrm{s}} \boldsymbol{u}, \boldsymbol{\tau}\right)+\frac{\lambda}{2 \mu}\left(\boldsymbol{a} \cdot \nabla \boldsymbol{\sigma}-\boldsymbol{\sigma} \cdot \nabla \boldsymbol{a}-(\nabla \boldsymbol{a})^{T} \cdot \boldsymbol{\sigma}, \boldsymbol{\tau}\right)
\end{aligned}
$$

To guarantee the well-posedness of problem (9), $\lambda$ must be small enough and the advection velocity must satisfy $\mathbf{H 1}$ below. Note that this assumption is consistent with those used in previous works [21,25,39], and it is obviously inherited by the discrete problem:

H1 $\boldsymbol{a} \in \mathcal{V}, \nabla \cdot \boldsymbol{a}=0,\|\boldsymbol{a}\|_{L^{\infty}(\Omega)} \leq M_{a}<\infty,\|\nabla \boldsymbol{a}\|_{L^{\infty}(\Omega)} \leq M_{g}<\infty$.

\subsection{Stability of the Galerkin finite element discretization}

Let us consider a finite element partition $\mathcal{P}_{h}=\{K\}$ of the domain $\Omega$ of diameter $h$. For simplicity, we will consider quasi-uniform refinements, and thus all the element diameters can be bounded above and below by constants multiplying $h$. Under the above considerations, we can construct conforming finite elements spaces, $\mathcal{V}_{h} \subset \mathcal{V}, \mathcal{Q}_{h} \subset \mathcal{Q}$ and $\boldsymbol{\Upsilon}_{h} \subset \boldsymbol{\Upsilon}$ in the usual manner. In practice, the zero mean for the pressure space can be replaced by prescribing the pressure at an arbitrary point, and the symmetry of the stress tensor 
can be forced by choosing the degrees of freedom corresponding to the upper diagonal of this tensor. The condition that the convective derivative of the stress be square integrable will follow from $\mathbf{H} \mathbf{1}$ and choosing the stresses continuous, for example. The advection velocity $\boldsymbol{a}$ will be approximated by $\boldsymbol{a}_{h} \in \mathcal{V}_{h}$; to simplify the exposition, we will consider that this approximation also satisfies $\mathbf{H 1}$ (the zero divergence condition could be avoided by the usual modification of the convective term in its skew-symmetric form).

If $\boldsymbol{\mathcal { X }}_{h}=\mathcal{V}_{h} \times \mathcal{Q}_{h} \times \boldsymbol{\Upsilon}_{h}$, and $\boldsymbol{U}_{h}=\left[\boldsymbol{u}_{h}, p_{h}, \boldsymbol{\sigma}_{h}\right]$, the Galerkin finite element approximation consists in finding $\boldsymbol{U}_{h} \in \mathcal{X}_{h}$ such that

$$
B\left(\boldsymbol{U}_{h}, \boldsymbol{V}_{h}\right)=\left\langle\boldsymbol{f}, \boldsymbol{v}_{h}\right\rangle
$$

for all $\boldsymbol{V}_{h}=\left[\boldsymbol{v}_{h}, q_{h}, \boldsymbol{\tau}_{h}\right] \in \boldsymbol{X}_{h}$.

At the moment, we have posed no restrictions on the choice of the finite element spaces. However, there are restriction that must be satisfied. For example, using the fact that $\boldsymbol{a}_{h}$ is divergence free, it is readily checked that

$$
B\left(\boldsymbol{U}_{h},\left[(1-\beta) \boldsymbol{u}_{h},(1-\beta) p_{h}, \boldsymbol{\sigma}_{h}\right]\right)=2 \beta \mu(1-\beta)\left\|\nabla^{\mathrm{s}} \boldsymbol{u}_{h}\right\|^{2}+\frac{1}{2 \mu}\left\|\boldsymbol{\sigma}_{h}\right\|^{2}-\frac{\lambda}{2 \mu}\left(\boldsymbol{\sigma}_{h} \cdot \nabla \boldsymbol{a}_{h}+\left(\nabla \boldsymbol{a}_{h}\right)^{T} \cdot \boldsymbol{\sigma}_{h}, \boldsymbol{\sigma}_{h}\right)
$$

Assuming $\lambda \nabla \boldsymbol{a}_{h}$ to be small enough, this expression provides only control on $\left\|\boldsymbol{\sigma}_{h}\right\|^{2}$ for all $\beta \in(0,1)$. To control the other two fields one has then to make use of the two inf-sup conditions that restrict the possible interpolations:

to control $p_{h}$, and

$$
\inf _{q_{h} \in \mathcal{Q}_{h}} \sup _{\boldsymbol{v}_{h} \in \mathcal{V}_{h}} \frac{\left(q_{h}, \nabla \cdot \boldsymbol{v}_{h}\right)}{\left\|\boldsymbol{v}_{h}\right\|_{\mathcal{V}_{h}}\left\|q_{h}\right\|_{\mathcal{Q}_{h}}} \geq C_{1}
$$

$$
\inf _{\boldsymbol{v}_{h} \in \mathcal{V}_{h} \boldsymbol{\tau}_{h} \in \boldsymbol{\Upsilon}_{h}} \frac{\left(\boldsymbol{\tau}_{h}, \nabla^{\mathrm{s}} \boldsymbol{v}_{h}\right)}{\left\|\boldsymbol{\tau}_{h}\right\|_{\boldsymbol{\Upsilon}_{h}}\left\|\boldsymbol{v}_{h}\right\|_{\mathcal{V}_{h}}} \geq C_{2}
$$

to control $\nabla^{\mathrm{s}} \boldsymbol{u}_{h}$, where $C_{1}$ and $C_{2}$ are positive constants. It is therefore required that the finite element spaces satisfy (12)-(13). These two conditions pose stringent requirements on the choice of the finite element spaces (see for example [34] for the 2D case and [6] for the 3D case). Our intention in this paper is to analyze a stabilized finite element formulation that avoids the need for such conditions and, in particular, allows one to use equal interpolation for all the unknowns, including the possibility to use discontinuous interpolations.

Let us introduce some notation. Summation over all the elements of $\mathcal{P}_{h}$ will be indicated as $\sum_{K}$. The collection of all edges (faces, for $d=3$ ) will be denoted by $\mathcal{E}_{h}=\{E\}$ and, as for the elements, summation over all these edges will be indicated as $\sum_{E}$. Suppose now that elements $K_{1}$ and $K_{2}$ share an edge $E$, and let $\boldsymbol{n}_{1}$ and $\boldsymbol{n}_{2}$ be the normals to $E$ exterior to $K_{1}$ and $K_{2}$, respectively. For a scalar function $g$, possibly discontinuous across $E$, we define its jump as $\llbracket \boldsymbol{n} g \rrbracket_{E}:=\left.\boldsymbol{n}_{1} g\right|_{\partial K_{1} \cap E}+\left.\boldsymbol{n}_{2} g\right|_{\partial K_{2} \cap E}$, and for a vector or tensor $\boldsymbol{v}, \llbracket \boldsymbol{n} \cdot \boldsymbol{v} \rrbracket_{E}:=\left.\boldsymbol{n}_{1} \cdot \boldsymbol{v}\right|_{\partial K_{1} \cap E}+\left.\boldsymbol{n}_{2} \cdot \boldsymbol{v}\right|_{\partial K_{2} \cap E}$. When $E \subset \partial \Omega$ and $\boldsymbol{n}$ is the external normal, these definitions reduce to $\llbracket \boldsymbol{n} g \rrbracket_{E}:=\left.\boldsymbol{n} g\right|_{E}$ and $\llbracket \boldsymbol{n} \cdot \boldsymbol{v} \rrbracket_{E}:=\left.\boldsymbol{n} \cdot \boldsymbol{v}\right|_{E}$. Generic positive constants will be denoted by $C$, possibly with subscripts and with different values in different appearances. The symbol $\lesssim$ will be used for $\leq$ up to constants.

\section{Stabilized Finite ElEMENT METHod}

In this section we summarize the stabilized formulation analyzed. This formulation was proposed in [13] and tested numerically in $[11,13,14]$, but the numerical analysis was not performed yet. As a novelty of this 
work, we will introduce an additional stabilizing term for the subscales on the element boundaries that the original work does not include, which allows us to consider discontinuous pressure and stress interpolations.

The method consist in replacing (11) by the following problem: find $\boldsymbol{U}_{h} \in \boldsymbol{X}_{h}$ such that

$$
B_{\text {stab }}\left(\boldsymbol{U}_{h}, \boldsymbol{V}_{h}\right)=B\left(\boldsymbol{U}_{h}, \boldsymbol{V}_{h}\right)+B^{*}\left(\boldsymbol{U}_{h}, \boldsymbol{V}_{h}\right)=\left\langle\boldsymbol{f}, \boldsymbol{v}_{h}\right\rangle
$$

for all $\boldsymbol{V}_{h} \in \mathcal{X}_{h}$, where $B^{*}$ represent the stabilizing part of the model, defined as

$$
B^{*}\left(\boldsymbol{U}_{h}, \boldsymbol{V}_{h}\right)=S_{1}^{\perp}\left(\boldsymbol{U}_{h}, \boldsymbol{V}_{h}\right)+S_{2}^{\perp}\left(\boldsymbol{U}_{h}, \boldsymbol{V}_{h}\right)+S_{3}^{\perp}\left(\boldsymbol{U}_{h}, \boldsymbol{V}_{h}\right)
$$

where the three additional terms that define $B^{*}\left(\boldsymbol{U}_{h}, \boldsymbol{V}_{h}\right)$ are associated to the stabilization terms of each equation and are defined as

$$
\begin{aligned}
& S_{1}^{\perp}\left(\boldsymbol{U}_{h}, \boldsymbol{V}_{h}\right)=\sum_{K} \alpha_{u}\left\langle P_{u}^{\perp}\left(\rho \boldsymbol{a}_{h} \cdot \nabla \boldsymbol{u}_{h}\right), P_{h}^{\perp}\left(\rho \boldsymbol{a}_{h} \cdot \nabla \boldsymbol{v}_{h}\right)\right\rangle_{K}+\sum_{K} \alpha_{u}\left\langle P_{u}^{\perp}\left(\nabla p_{h}\right), P_{h}^{\perp}\left(\nabla q_{h}\right)\right\rangle_{K} \\
& \quad+(1-\beta) \sum_{K} \alpha_{u}\left\langle P_{u}^{\perp}\left(\nabla \cdot \boldsymbol{\sigma}_{h}\right), P_{u}^{\perp}\left(\nabla \cdot \boldsymbol{\tau}_{h}\right)\right\rangle_{K} \\
& \quad+\sum_{E} \alpha_{[u]}\left\langle\llbracket\left(\boldsymbol{n} q_{h}-(1-\beta) \boldsymbol{n} \cdot \boldsymbol{\tau}_{h}\right)+2 \beta \mu \boldsymbol{n} \cdot \nabla^{\mathrm{s}} \boldsymbol{v}_{h} \rrbracket, \llbracket\left(\boldsymbol{n} p_{h}-\boldsymbol{n} \cdot \boldsymbol{\sigma}_{h}\right)-2 \beta \mu \boldsymbol{n} \cdot \nabla^{\mathrm{s}} \boldsymbol{u}_{h} \rrbracket\right\rangle_{E} \\
& S_{2}^{\perp}\left(\boldsymbol{U}_{h}, \boldsymbol{V}_{h}\right)=\sum_{K} \alpha_{p}\left\langle P_{p}^{\perp}\left(\nabla \cdot \boldsymbol{u}_{h}\right), P_{p}^{\perp}\left(\nabla \cdot \boldsymbol{v}_{h}\right)\right\rangle_{K}, \\
& S_{3}^{\perp}\left(\boldsymbol{U}_{h}, \boldsymbol{V}_{h}\right)=\sum_{K} \alpha_{\sigma}\left\langle P_{\sigma}^{\perp}\left(\boldsymbol{R}_{\sigma}\right), P_{\sigma}^{\perp}\left(\nabla^{\mathrm{s}} \boldsymbol{v}_{h}-\frac{\lambda}{2 \mu}\left(\boldsymbol{a}_{h} \cdot \nabla \boldsymbol{\tau}_{h}+\boldsymbol{\tau}_{h} \cdot\left(\nabla \boldsymbol{a}_{h}\right)^{T}+\nabla \boldsymbol{a}_{h} \cdot \boldsymbol{\tau}_{h}\right)\right)\right\rangle_{K}
\end{aligned}
$$

In the last expression, $\boldsymbol{\mathcal { R }}_{\sigma}$ represents the residual of the constitutive equation without the stress, given by

$$
\boldsymbol{\mathcal { R }}_{\sigma}=(1-\beta) \nabla^{\mathrm{s}} \boldsymbol{u}_{h}-\frac{\lambda}{2 \mu}\left(\boldsymbol{a}_{h} \cdot \nabla \boldsymbol{\sigma}_{h}-\boldsymbol{\sigma}_{h} \cdot \nabla \boldsymbol{a}_{h}-\left(\nabla \boldsymbol{a}_{h}\right)^{T} \cdot \boldsymbol{\sigma}_{h}\right)
$$

In the numerical analysis below we will also use the notation

$$
\boldsymbol{\sigma}_{h} \cdot \nabla \boldsymbol{a}_{h}+\left(\nabla \boldsymbol{a}_{h}\right)^{T} \cdot \boldsymbol{\sigma}_{h}=\dot{\boldsymbol{\sigma}}_{h}^{*}+\dot{\boldsymbol{\sigma}}_{h}^{* *} \text { and } \boldsymbol{\sigma}_{h} \cdot\left(\nabla \boldsymbol{a}_{h}\right)^{T}+\nabla \boldsymbol{a}_{h} \cdot \boldsymbol{\sigma}_{h}=\dot{\boldsymbol{\sigma}}_{h}^{*}-\dot{\boldsymbol{\sigma}}_{h}^{* *}
$$

where $\dot{\boldsymbol{\sigma}}_{h}^{*}=\boldsymbol{\sigma}_{h} \cdot \nabla^{\mathrm{s}} \boldsymbol{a}_{h}+\nabla^{\mathrm{s}} \boldsymbol{a}_{h} \cdot \boldsymbol{\sigma}_{h}$ and $\dot{\boldsymbol{\sigma}}_{h}^{* *}=\boldsymbol{\sigma}_{h} \cdot \nabla^{\mathrm{as}} \boldsymbol{a}_{h}-\nabla^{\mathrm{as}} \boldsymbol{a}_{h} \cdot \boldsymbol{\sigma}_{h}$. In these expressions, $\nabla^{\text {as }} \boldsymbol{a}_{h}$ represents the skew-symmetric part of the velocity gradient, given by

$$
\nabla^{\mathrm{as}} \boldsymbol{a}=\frac{1}{2}\left[\nabla \boldsymbol{a}-(\nabla \boldsymbol{a})^{T}\right]
$$

In (15)-(17), $P_{u}^{\perp}$ represents the projection $L^{2}$-orthogonal to the velocity space without boundary conditions, $P_{p}^{\perp}$ the projection $L^{2}$-orthogonal to the pressure space and $P_{\sigma}^{\perp}$ the projection $L^{2}$-orthogonal to the stress space. For example $P_{u}^{\perp}=I-P_{u}$, where $P_{u}$ is the $L^{2}$ projection onto the velocity finite element space without boundary conditions. We will also need to use the $L^{2}$-projection onto the velocity space with boundary conditions, $\mathcal{V}_{h}$, that we will denote by $P_{u, 0}$. The last term in $S_{1}^{\perp}$ is an approximation to the subscales on the element boundaries and allows us to consider discontinuous interpolations for the pressure 
and the stress. The design of the stabilization terms without the boundary term was presented in [13] for the stationary three-field viscoelastic case. For the design of the boundary term we refer to [18], where a three-field Stokes problem was analyzed in detail. The extension to the Oldroyd-B viscoelastic case is trivial.

The stabilization parameters $\alpha_{i}, i=u$ or $i=p$ or $i=\sigma$, are computed within each element $K$ as

$$
\begin{aligned}
\alpha_{u} & =\left(c_{1} \frac{\mu}{h^{2}}+c_{2} \frac{\rho\left|\boldsymbol{a}_{h}\right|}{h}\right)^{-1} \\
\alpha_{p} & =\frac{h^{2}}{c_{1} \alpha_{1}} \\
\alpha_{\sigma} & =\left(c_{3} \frac{1}{2 \mu}+c_{4} \frac{\lambda}{2 \mu} \frac{\left|\boldsymbol{a}_{h}\right|}{h}+c_{5} \frac{\lambda}{\mu}\left|\nabla \boldsymbol{a}_{h}\right|\right)^{-1}
\end{aligned}
$$

A possible justification for these parameters is the Fourier analysis presented in [18] for the three-field Stokes problem or in [17] for the transient Navier-Stokes problem. The constants $c_{i}, i=1,2,3,4,5$, are algorithmic parameters. As a reference, the values used in the numerical experiments in $[11,13,14]$ for linear elements were $c_{1}=4.0$ or $12.0, c_{2}=2.0, c_{3}=4.0$ and $c_{4}=c_{5}=0.25$ or 1.0. For higher order elements these values should be modified in terms of the interpolation order used (more details can be found in the numerical references where the method was tested).

The boundary stabilization parameter in $S_{1}^{\perp}$ can be defined as $\alpha_{[u]}=\frac{\delta_{0} h}{2 \mu}$, as in $[18,20]$, where $\delta_{0}$ is an algorithmic parameter that can be taken as $\delta_{0}=0.1$.

The method presented is a mix of an orthogonal term-by-term formulation for the momentum and continuity equations and a residual-based formulation for the constitutive equation. The term-by-term part is not just a simplification of a standard residual based one. For smooth solutions, both have an optimal convergence rate in $h$. However, in problems where the solution has strong gradients, we have found the term-by-term formulation more robust than the residual-based one, which can be explained using numerical analysis as we will show below. The method we analyze permits a term-by-term control of the orthogonal projections of the pressure gradient, the convective term of the momentum equation and the divergence of the elastic stress which is not possible using the residual-based formulation.

\section{Numerical Analysis}

The numerical analysis performed in this section follows a more or less standard approach. First we prove stability in the form of inf-sup condition in a mesh dependent norm that depends on the stabilized formulation used. Next we prove convergence in the same norm. Stability and convergence in natural norms, that is

to say, the norms where the continuous problem is posed, are then proved. Finally, we obtain an $L^{2}$-error estimate for the velocity using a duality argument.

\subsection{Preliminaries}

As it has been mentioned in Section 3, we will consider for the sake of conciseness quasi-uniform finite element partitions. Therefore, we assume that there is a constant $c_{\text {inv }}$, independent of the mesh size $h$ (the maximum of all the element diameters), such that

$$
\left\|\nabla v_{h}\right\|_{K} \leq c_{\mathrm{inv}} h^{-1}\left\|v_{h}\right\|_{K}
$$


for all finite element functions $v_{h}$ defined on $K \in \mathcal{P}_{h}$, which can be either scalars, vectors or tensors. Similarly, the trace inequality

$$
\|v\|_{\partial K}^{2} \leq c_{\mathrm{tr}}\left(h^{-1}\|v\|_{K}^{2}+h\|\nabla v\|_{K}^{2}\right)
$$

is assumed to hold for functions $v \in H^{1}(K), K \in \mathcal{P}_{h}$. The last term can be dropped if $v$ is a polynomial on the element domain $K$. Thus, if $\varphi_{h}$ is a piecewise discontinuous polynomial and $\psi_{h}$ a continuous one, it follows that

$$
\sum_{E}\left\|\llbracket \boldsymbol{n} \varphi_{h} \rrbracket\right\|_{E}^{2} \leq 2 c_{\mathrm{tr}} h^{-1} \sum_{K}\left\|\varphi_{h}\right\|_{K}^{2}, \quad \sum_{E}\left\|\psi_{h}\right\|_{E}^{2} \leq \frac{c_{\mathrm{tr}}}{2} h^{-1} \sum_{K}\left\|\psi_{h}\right\|_{K}^{2}
$$

We will also make use of Korn's inequality, which holds for the conforming approximation that we consider:

$$
\left\|\boldsymbol{v}_{h}\right\|_{H^{1}(\Omega)}^{2} \leq c\left\|\nabla^{\mathrm{s}} \boldsymbol{v}_{h}\right\|^{2}, \quad \text { with } \boldsymbol{v}_{h}=\mathbf{0} \text { on } \partial \Omega
$$

Let $\mathcal{W}_{h}$ be a finite element space of degree $k_{v}$. For any function $v \in H^{k_{v}^{\prime}+1}(\Omega)$ and for $i=0,1$, we define the interpolation errors $\varepsilon_{i}(v)$ from the interpolation estimates

$$
\inf _{v_{h} \in \mathcal{W}_{h}} \sum_{K}\left\|v-v_{h}\right\|_{H^{i}(K)} \leq C h^{k_{v}^{\prime \prime}+1-i} \sum_{K}\|v\|_{H^{k_{v}^{\prime \prime}+1}(K)}=: \sum_{K} \varepsilon_{i, K}(v)=: \varepsilon_{i}(v)
$$

where $k_{v}^{\prime \prime}=\min \left(k_{v}, k_{v}^{\prime}\right)$. We will denote by $\tilde{v}_{h}$ the best approximation of $v$ in $\mathcal{W}_{h}$. Clearly, we may take $\varepsilon_{0}(v)=h \varepsilon_{1}(v)$.

We will need a condition on the interpolating spaces that holds in the case of equal order interpolations (see [19]), and that can be written as follows:

H2 Given $\boldsymbol{a}_{h}, \boldsymbol{v}_{h} \in \mathcal{V}_{h}, q_{h} \in \mathcal{Q}_{h}, \boldsymbol{\tau}_{h} \in \boldsymbol{\Upsilon}_{h}$ and $\boldsymbol{z}_{h}:=\rho \boldsymbol{a}_{h} \cdot \nabla \boldsymbol{v}_{h}+\nabla q_{h}-\nabla \cdot \boldsymbol{\tau}_{h}$, there holds

$$
\left\|\boldsymbol{z}_{h}\right\| \leq c_{\mathrm{m}}\left(\left\|P_{u, 0}\left(\boldsymbol{z}_{h}\right)\right\|+\left\|P_{u}^{\perp}\left(\boldsymbol{z}_{h}\right)\right\|\right)
$$

for a constant $c_{\mathrm{m}}>0$.

According to this condition, the component of $P_{u}\left(\boldsymbol{z}_{h}\right)$ that corresponds to the boundary of $\Omega$ can be bounded in terms of the right-hand-side of Eq. (22). To prove this, one can use the macro-element technique employed in [19].

\subsection{Stability and convergence in a mesh-dependent norm}

The norm in which the results will be first presented is

$$
\begin{aligned}
\left\|\boldsymbol{V}_{h}\right\|_{W}^{2}= & 2 \beta \mu(1-\beta) \sum_{K}\left\|\nabla^{\mathrm{s}} \boldsymbol{v}_{h}\right\|_{K}^{2}+\frac{1}{2 \mu} \sum_{K}\left\|\boldsymbol{\tau}_{h}\right\|_{K}^{2}+\sum_{K} \alpha_{u}\left\|\rho \boldsymbol{a}_{h} \cdot \nabla \boldsymbol{v}_{h}+\nabla q_{h}-\nabla \cdot \boldsymbol{\tau}_{h}\right\|_{K}^{2} \\
& +\sum_{K} \alpha_{u}\left\|P_{u}^{\perp}\left(\rho \boldsymbol{a}_{h} \cdot \nabla \boldsymbol{v}_{h}\right)\right\|_{K}^{2}+(1-\beta) \sum_{K} \alpha_{u}\left\|P_{u}^{\perp}\left(\nabla \cdot \boldsymbol{\tau}_{h}\right)\right\|_{K}^{2}+\sum_{K} \alpha_{u}\left\|P_{u}^{\perp}\left(\nabla q_{h}\right)\right\|_{K}^{2} \\
& +\sum_{K} \alpha_{p}\left\|\nabla \cdot \boldsymbol{v}_{h}\right\|_{K}^{2}+\sum_{K} \alpha_{\sigma}\left\|-(1-\beta) \nabla^{\mathrm{s}} \boldsymbol{v}_{h}+\frac{\lambda}{2 \mu}\left(\boldsymbol{a}_{h} \cdot \nabla \boldsymbol{\tau}_{h}-\dot{\boldsymbol{\tau}}_{h}^{* *}\right)\right\|_{K}^{2}
\end{aligned}
$$




$$
+(1-\beta) \sum_{E} \alpha_{[u]}\left\|\llbracket \boldsymbol{n} q_{h}-\boldsymbol{n} \cdot \boldsymbol{\tau}_{h} \rrbracket\right\|_{E}^{2}
$$

although later on we will transform our results to natural norms under the assumption of small elasticity and large viscosity. Note that the term multiplied by $\alpha_{p}$ is unnecessary, since it is already contained in the first right hand side term. However, we will keep it to see the effect of the subscale associated to the pressure. It can also be helpful for some non-conforming elements (not considered in this work) for which the discrete Korn inequality does not hold (see e.g. [30]).

If $\beta$ is very small (or $\beta=0$ ) the control on velocity gradient can be obtained from the term multiplied by $\alpha_{\sigma}$. However, to simplify a little the analysis we will consider $\beta>0$, and the stability provided by the first term relevant enough.

Note that the stabilization term $S_{1}^{\perp}$ allows us to have a term-by-term control of the orthogonal projections of the convective term, the pressure gradient and the divergence of the elastic stress tensor. This fact may be an explanation of why we have found the term-by-term stabilization more robust than the residual based one in the presence of high gradients.

Our first result states the formulation we consider is stable in the working norm (23):

Theorem 1 (Stability). Suppose that H2 holds. For $\lambda$ small enough, there is a constant $C>0$ such that

$$
\inf _{\boldsymbol{U}_{h} \in \mathcal{X}_{h}} \sup _{\boldsymbol{V}_{h} \in \mathcal{X}_{h}} \frac{B_{\text {stab }}\left(\boldsymbol{U}_{h}, \boldsymbol{V}_{h}\right)}{\left\|\boldsymbol{U}_{h}\right\|_{W}\left\|\boldsymbol{V}_{h}\right\|_{W}} \geq C
$$

provided $\delta_{0}$ is taken small enough and the constants $c_{i}, i=1,2,3,4,5$ in (18)-(20) are large enough.

Proof. Given $\boldsymbol{U}_{h} \in \boldsymbol{X}_{h}$, consider $\boldsymbol{U}_{h 1}=\left((1-\beta) \boldsymbol{u}_{h},(1-\beta) p_{h}, \boldsymbol{\sigma}_{h}\right)$. Using only Schwarz's inequality we obtain

$$
\begin{aligned}
& B_{\text {stab }}\left(\boldsymbol{U}_{h}, \boldsymbol{U}_{h 1}\right) \geq 2 \beta(1-\beta) \mu\left\|\nabla^{\mathrm{s}} \boldsymbol{u}_{h}\right\|^{2}+\frac{1}{2 \mu} \sum_{K}\left(1-2 \lambda\left\|\nabla \boldsymbol{a}_{h}\right\|_{K}\right)\left\|\boldsymbol{\sigma}_{h}\right\|_{K}^{2} \\
& \quad-\frac{1}{2 \mu} \sum_{K}\left(4 \lambda\left(\alpha_{\sigma} \frac{\lambda}{2 \mu}\right)\left\|\nabla^{\mathrm{s}} \boldsymbol{a}_{h}\right\|_{K}^{2}\right)\left\|\boldsymbol{\sigma}_{h}\right\|_{K}^{2}+(1-\beta) \sum_{K} \alpha_{p}\left\|P_{p}^{\perp}\left(\nabla \cdot \boldsymbol{u}_{h}\right)\right\|_{K}^{2} \\
& +(1-\beta) \sum_{K} \alpha_{u}\left\|P_{u}^{\perp}\left(\rho \boldsymbol{a}_{h} \cdot \nabla \boldsymbol{u}_{h}\right)\right\|_{K}^{2}+(1-\beta) \sum_{K} \alpha_{u}\left\|P_{u}^{\perp}\left(\nabla p_{h}\right)\right\|_{K}^{2} \\
& \quad+(1-\beta) \sum_{K} \alpha_{u}\left\|P_{u}^{\perp}\left(\nabla \cdot \boldsymbol{\sigma}_{h}\right)\right\|_{K}^{2}+\sum_{K} \alpha_{\sigma}\left\|P_{\sigma}^{\perp}\left(-(1-\beta) \nabla^{\mathrm{s}} \boldsymbol{u}_{h}+\frac{\lambda}{2 \mu}\left(\boldsymbol{a}_{h} \cdot \nabla \boldsymbol{\sigma}_{h}-\dot{\boldsymbol{\sigma}}_{h}^{* *}\right)\right)\right\|_{K}^{2} \\
& \quad+(1-\beta) \sum_{E} \frac{\delta_{0} h}{2 \mu}\left\|\llbracket \boldsymbol{n} p_{h}-\boldsymbol{n} \cdot \boldsymbol{\sigma}_{h} \rrbracket\right\|_{E}^{2}-(1-\beta) \sum_{E} \frac{\delta_{0} h}{2 \mu}\left\|\llbracket 2 \beta \mu \boldsymbol{n} \cdot \nabla^{\mathrm{s}} \boldsymbol{u}_{h} \rrbracket\right\|_{E}^{2}
\end{aligned}
$$

Using now (21) we obtain

$$
\begin{gathered}
B_{\text {stab }}\left(\boldsymbol{U}_{h}, \boldsymbol{U}_{h 1}\right) \geq 2 \beta(1-\beta) \mu \sum_{K}\left(1-2 \beta \delta_{0} c_{\mathrm{tr}}\right)\left\|\nabla^{\mathrm{s}} \boldsymbol{u}_{h}\right\|_{K}^{2}+(1-\beta) \sum_{K} \alpha_{u}\left\|P_{u}^{\perp}\left(\rho \boldsymbol{a}_{h} \cdot \nabla \boldsymbol{u}_{h}\right)\right\|_{K}^{2} \\
+(1-\beta) \sum_{K} \alpha_{p}\left\|P_{p}^{\perp}\left(\nabla \cdot \boldsymbol{u}_{h}\right)\right\|_{K}^{2}+\frac{1}{2 \mu} \sum_{K}\left(1-2 \lambda\left\|\nabla \boldsymbol{a}_{h}\right\|_{K}-4 \lambda \alpha_{\sigma} \frac{\lambda}{2 \mu}\left\|\nabla^{\mathrm{s}} \boldsymbol{a}_{h}\right\|_{K}^{2}\right)\left\|\boldsymbol{\sigma}_{h}\right\|_{K}^{2}
\end{gathered}
$$




$$
\begin{aligned}
& +\alpha_{u}(1-\beta) \sum_{K}\left\|P_{u}^{\perp}\left(\nabla p_{h}\right)\right\|_{K}^{2}+(1-\beta) \sum_{K} \alpha_{u}\left\|P_{u}^{\perp}\left(\nabla \cdot \boldsymbol{\sigma}_{h}\right)\right\|_{K}^{2} \\
& \left.+\alpha_{\sigma}\left\|P_{\sigma}^{\perp}\left(-(1-\beta) \nabla^{\mathrm{s}} \boldsymbol{u}_{h}+\frac{\lambda}{2 \mu}\left(\boldsymbol{a}_{h} \cdot \nabla \boldsymbol{\sigma}_{h}-\dot{\boldsymbol{\sigma}}_{h}^{* *}\right)\right)\right\|_{K}^{2}+(1-\beta) \sum_{E} \frac{\delta_{0} h}{2 \mu} \| \llbracket \boldsymbol{n} p_{h}-\boldsymbol{n} \cdot \boldsymbol{\sigma}_{h}\right] \|_{E}^{2}
\end{aligned}
$$

An important restriction inherited from the continuous case is that the stability of the elastic stresses can be ensured only for small Weissenberg numbers. In the above expression the factor of the term $\left\|\boldsymbol{\sigma}_{h}\right\|^{2}$ must be strictly positive to guarantee stability. In this factor we have two negative components, the first $2 \lambda\left\|\nabla \boldsymbol{a}_{h}\right\|$ comes from the Galerkin method, and the second $4 \lambda \alpha_{\sigma} \frac{\lambda}{2 \mu}\left\|\nabla^{\mathrm{s}} \boldsymbol{a}_{h}\right\|^{2}$ from the stabilized formulation. All the analysis carried out assumes that

$$
1-2 \lambda\left\|\nabla \boldsymbol{a}_{h}\right\|_{K}-4 \lambda \alpha_{\sigma} \frac{\lambda}{2 \mu}\left\|\nabla^{\mathrm{s}} \boldsymbol{a}_{h}\right\|_{K}^{2} \geq C>0
$$

The basic idea to prove Theorem 1 is to obtain control on the components on the finite element space for the terms whose orthogonal component appears in the above expression. The key point is that this control comes from the Galerkin terms of $B_{\text {stab }}$ in (14).

Let us start considering $\boldsymbol{V}_{h 1}=\alpha_{u}(1-\beta)\left(P_{u, 0}\left(\rho \boldsymbol{a}_{h} \cdot \nabla \boldsymbol{u}_{h}+\nabla p_{h}-\nabla \cdot \boldsymbol{\sigma}_{h}\right), 0, \mathbf{0}\right)$. Recalling that $P_{u, 0}$ is defined based on elementwise integrals, $P_{u, 0}\left(\rho \boldsymbol{a}_{h} \cdot \nabla \boldsymbol{u}_{h}+\nabla p_{h}-\nabla \cdot \boldsymbol{\sigma}_{h}\right)$ is well defined. We will use the abbreviation $\boldsymbol{v}_{1} \equiv P_{u, 0}\left(\rho \boldsymbol{a}_{h} \cdot \nabla \boldsymbol{u}_{h}+\nabla p_{h}-\nabla \cdot \boldsymbol{\sigma}_{h}\right)$. Using Young's inequality, inverse estimates and (21), we obtain

$$
\begin{aligned}
& B_{\text {stab }}\left(\boldsymbol{U}_{h}, \boldsymbol{V}_{h 1}\right)=B\left(\boldsymbol{U}_{h}, \boldsymbol{V}_{h 1}\right)+B^{*}\left(\boldsymbol{U}_{h}, \boldsymbol{V}_{h 1}\right) \\
& \geq B^{*}\left(\boldsymbol{U}_{h}, \boldsymbol{V}_{h 1}\right)+(1-\beta)\left(1-\frac{\varepsilon_{1}}{2}-\frac{c_{\mathrm{tr}}}{2} \frac{\varepsilon_{2}}{2}\right) \sum_{K} \alpha_{u}\left\|P_{u, 0}\left(\rho \boldsymbol{a}_{h} \cdot \nabla \boldsymbol{u}_{h}+\nabla p_{h}-\nabla \cdot \boldsymbol{\sigma}_{h}\right)\right\|_{K}^{2} \\
& -\mu(1-\beta)(2 \beta)^{2} \frac{c_{\mathrm{inv}}^{2}}{2 \varepsilon_{1}} \sum_{K} \alpha_{u} \frac{\mu}{h^{2}}\left\|\nabla^{\mathrm{s}} \boldsymbol{u}_{h}\right\|_{K}^{2} \\
& -(1-\beta) \frac{1}{2 \varepsilon_{2}} \sum_{E} \frac{\alpha_{u}}{h}\left\|\llbracket\left(\boldsymbol{n} p_{h}-\boldsymbol{n} \cdot \boldsymbol{\sigma}_{h}\right)-2 \beta \mu \boldsymbol{n} \cdot \nabla^{\mathrm{s}} \boldsymbol{u}_{h} \rrbracket\right\|_{E}^{2} \\
& \geq B^{*}\left(\boldsymbol{U}_{h}, \boldsymbol{V}_{h 1}\right)+(1-\beta)\left(1-\frac{\varepsilon_{1}}{2}-\frac{c_{\mathrm{tr}}}{2} \frac{\varepsilon_{2}}{2}\right) \sum_{K} \alpha_{u}\left\|P_{u, 0}\left(\rho \boldsymbol{a}_{h} \cdot \nabla \boldsymbol{u}_{h}+\nabla p_{h}-\nabla \cdot \boldsymbol{\sigma}_{h}\right)\right\|_{K}^{2} \\
& -\mu(1-\beta)(2 \beta)^{2}\left(\frac{c_{\mathrm{inv}}^{2}}{2 \varepsilon_{1}}+4 \frac{c_{\mathrm{tr}}}{2 \varepsilon_{2}}\right) \sum_{K} \alpha_{u} \frac{\mu}{h^{2}}\left\|\nabla^{\mathrm{s}} \boldsymbol{u}_{h}\right\|_{K}^{2} \\
& -2(1-\beta) \frac{1}{2 \varepsilon_{2}} \sum_{E} \frac{\alpha_{u}}{h}\left\|\llbracket \boldsymbol{n} p_{h}-\boldsymbol{n} \cdot \boldsymbol{\sigma}_{h} \rrbracket\right\|_{E}^{2}
\end{aligned}
$$

for any $\varepsilon_{1}, \varepsilon_{2}$. On the other hand, the stabilizing part leads to

$$
\begin{aligned}
& B^{*}\left(\boldsymbol{U}_{h}, \boldsymbol{V}_{h 1}\right) \geq-(1-\beta) \frac{1}{2 \varepsilon_{4}} \sum_{K}\left(\alpha_{u}^{2} \frac{\rho\left\|\boldsymbol{a}_{h}\right\|_{K}}{h}\right)\left\|P_{u}^{\perp}\left(\rho \boldsymbol{a}_{h} \cdot \nabla \boldsymbol{u}_{h}\right)\right\|_{K}^{2} \\
& \quad-(1-\beta)\left(c_{\mathrm{inv}}\right)^{2} \sum_{K} \alpha_{u}^{2}\left(\frac{\alpha_{p}}{h^{2}} \frac{\varepsilon_{3}}{2}+\frac{\rho\left\|\boldsymbol{a}_{h}\right\|_{K}}{h} \frac{\varepsilon_{4}}{2}+\frac{2 \mu}{h^{2}} \beta^{2} \delta_{0} c_{\mathrm{tr}} \frac{\varepsilon_{5}}{2}+\frac{\alpha_{\sigma}}{h^{2}} \frac{\varepsilon_{6}}{2}\right)\left\|\boldsymbol{v}_{1}\right\|_{K}^{2}
\end{aligned}
$$




$$
\begin{aligned}
& -(1-\beta) \frac{1}{2 \varepsilon_{3}} \sum_{K} \alpha_{p}\left\|P_{p}^{\perp}\left(\nabla \cdot \boldsymbol{u}_{h}\right)\right\|_{K}^{2} \\
& -2(1-\beta) \frac{1}{2 \varepsilon_{6}} \sum_{K} \alpha_{\sigma}\left\|P_{\sigma}^{\perp}\left(-(1-\beta) \nabla^{\mathrm{s}} \boldsymbol{u}_{h}+\frac{\lambda}{2 \mu}\left(\boldsymbol{a}_{h} \cdot \nabla \boldsymbol{\sigma}_{h}-\dot{\boldsymbol{\sigma}}_{h}^{* *}\right)\right)\right\|_{K}^{2} \\
& -2(1-\beta) \frac{1}{2 \varepsilon_{6}}\left(\frac{\lambda}{2 \mu}\right)^{2} \sum_{K} \alpha_{\sigma}\left\|\dot{\boldsymbol{\sigma}}_{h}^{*}\right\|_{K}^{2} \\
& -2(1-\beta) \frac{1}{2 \varepsilon_{5}} \sum_{E} \alpha_{[u]}\left\|\llbracket \boldsymbol{n} p_{h}-\boldsymbol{n} \cdot \boldsymbol{\sigma}_{h} \rrbracket\right\|_{E}^{2}-8 \beta^{2}(1-\beta) \mu c_{\mathrm{tr}} \frac{1}{2 \varepsilon_{5}} \sum_{K} \delta_{0}\left\|\nabla^{\mathrm{s}} \boldsymbol{u}_{h}\right\|_{K}^{2}
\end{aligned}
$$

for any $\varepsilon_{i}, i=3,4,5,6$. Using $(26)$ in $(25)$ we get

$$
\begin{aligned}
& B_{\text {stab }}\left(\boldsymbol{U}_{h}, \boldsymbol{V}_{h 1}\right) \geq(1-\beta) \sum_{K} \alpha_{u} C_{u}\left\|\boldsymbol{v}_{1}\right\|_{K}^{2} \\
& -\mu(1-\beta)(2 \beta)^{2}\left(\frac{c_{\mathrm{inv}}^{2}}{2 \varepsilon_{1}}+4 \frac{c_{\mathrm{tr}}}{2 \varepsilon_{2}}+2 \frac{c_{\mathrm{tr}} \delta_{0}}{2 \varepsilon_{5}}\right) \sum_{K} \alpha_{u} \frac{\mu}{h^{2}}\left\|\nabla^{\mathrm{s}} \boldsymbol{u}_{h}\right\|_{K}^{2} \\
& -(1-\beta) \frac{1}{2 \varepsilon_{3}} \sum_{K} \alpha_{p}\left\|P_{p}^{\perp}\left(\nabla \cdot \boldsymbol{u}_{h}\right)\right\|_{K}^{2}-(1-\beta) \frac{1}{2 \varepsilon_{4}} \sum_{K} \alpha_{u}^{2} \frac{\rho\left\|\boldsymbol{a}_{h}\right\|_{K}}{h}\left\|P_{u}^{\perp}\left(\rho \boldsymbol{a}_{h} \cdot \nabla \boldsymbol{u}_{h}\right)\right\|_{K}^{2} \\
& -(1-\beta) 2 \frac{1}{2 \varepsilon_{6}} \sum_{K} \alpha_{\sigma}\left\|P_{\sigma}^{\perp}\left(-(1-\beta) \nabla^{\mathrm{s}} \boldsymbol{u}_{h}+\frac{\lambda}{2 \mu}\left(\boldsymbol{a}_{h} \cdot \nabla \boldsymbol{\sigma}_{h}-\dot{\boldsymbol{\sigma}}_{h}^{* *}\right)\right)\right\|_{K}^{2} \\
& -\frac{1}{2 \mu} 2(1-\beta) \frac{1}{2 \varepsilon_{6}} \sum_{K} \frac{\alpha_{\sigma}}{2 \mu}\left(\lambda\left\|\nabla^{\mathrm{s}} \boldsymbol{a}_{h}\right\|_{K}\right)^{2}\left\|\boldsymbol{\sigma}_{h}\right\|_{K}^{2} \\
& -2(1-\beta)\left(\frac{\delta_{0} h}{\mu}\left(\alpha_{u} \frac{\mu}{\delta_{0} h^{2}} \frac{1}{2 \varepsilon_{2}}+\frac{1}{2 \varepsilon_{5}}\right)\right) \sum_{E}\left\|\llbracket \boldsymbol{n} p_{h}-\boldsymbol{n} \cdot \boldsymbol{\sigma}_{h} \rrbracket\right\|_{E}^{2}
\end{aligned}
$$

with

$$
C_{u}:=1-\frac{\varepsilon_{1}}{2}-\frac{\varepsilon_{2}}{4} c_{\mathrm{tr}}-\alpha_{u}\left(\frac{\alpha_{p}}{h^{2}} \frac{\varepsilon_{3}}{2}+\frac{\rho\left\|\boldsymbol{a}_{h}\right\|_{K}}{h} \frac{\varepsilon_{4}}{2}+\frac{2 \mu}{h^{2}} \beta^{2} \delta_{0} c_{\mathrm{tr}} \frac{\varepsilon_{5}}{2}+\frac{\alpha_{\sigma}}{h^{2}} \frac{\varepsilon_{6}}{2}\right) c_{\mathrm{inv}}^{2}
$$

Let us consider now the test function $\boldsymbol{V}_{h 2}=\alpha_{p}(1-\beta)\left(\mathbf{0}, P_{p}\left(\nabla \cdot \boldsymbol{u}_{h}\right), \mathbf{0}\right)$. We will use the abbreviation $q_{2} \equiv P_{p}\left(\nabla \cdot \boldsymbol{u}_{h}\right)$. Proceeding as before we get

$$
\begin{aligned}
& B_{\text {stab }}\left(\boldsymbol{U}_{h}, \boldsymbol{V}_{h 2}\right) \geq(1-\beta) \sum_{K} \alpha_{p} C_{p}\left\|P_{p}\left(\nabla \cdot \boldsymbol{u}_{h}\right)\right\|_{K}^{2} \\
& -(1-\beta) \frac{\varepsilon_{7}}{2} \sum_{K} \alpha_{u}\left\|P_{u}^{\perp}\left(\nabla p_{h}\right)\right\|_{K}^{2}-2 \frac{\delta_{0} h}{2 \mu}(1-\beta) \varepsilon_{8} \sum_{E} \frac{\alpha_{p}}{\mu}\left\|\llbracket \boldsymbol{n} p_{h}-\boldsymbol{n} \cdot \boldsymbol{\sigma}_{h} \rrbracket\right\|_{E}^{2} \\
& -2 \frac{\delta_{0} h}{2 \mu}(1-\beta)(2 \beta \mu)^{2} \frac{2 c_{\mathrm{tr}}}{h} \varepsilon_{8} \sum_{K} \frac{\alpha_{p}}{\mu}\left\|\nabla^{\mathrm{s}} \boldsymbol{u}_{h}\right\|_{K}^{2}
\end{aligned}
$$

for any $\varepsilon_{7}, \varepsilon_{8}$, with

$$
C_{p}:=1-\frac{c_{\mathrm{inv}}^{2}}{2 \varepsilon_{7}} \frac{\alpha_{u} \alpha_{p}}{h^{2}}-\frac{\delta_{0} c_{\mathrm{tr}}}{2 \varepsilon_{8}}
$$


The last step is to consider as test function $\boldsymbol{V}_{h 3}=\alpha_{\sigma}\left(\mathbf{0}, 0, \boldsymbol{\sigma}_{3}\right)$, with

$$
\boldsymbol{\sigma}_{3}:=P_{\sigma}\left(-(1-\beta) \nabla^{\mathrm{s}} \boldsymbol{u}_{h}+\frac{\lambda}{2 \mu}\left(\boldsymbol{a}_{h} \cdot \nabla \boldsymbol{\sigma}_{h}-\boldsymbol{\sigma}_{h} \cdot \nabla \boldsymbol{a}_{h}-\left(\nabla \boldsymbol{a}_{h}\right)^{T} \cdot \boldsymbol{\sigma}_{h}\right)\right)
$$

Using the same tools as in the previous cases we now obtain

$$
\begin{aligned}
& B_{\text {stab }}\left(\boldsymbol{U}_{h}, \boldsymbol{V}_{h 3}\right) \geq-\frac{1}{2 \mu} \frac{1}{2 \varepsilon_{9}}\left\|\boldsymbol{\sigma}_{h}\right\|^{2}-2 \frac{\delta_{0} h}{2 \mu} \frac{1}{2 \varepsilon_{12}}(1-\beta) \sum_{E}\left\|\llbracket \boldsymbol{n} p_{h}-\boldsymbol{n} \cdot \boldsymbol{\sigma}_{h} \rrbracket\right\|_{E}^{2} \\
& -(1-\beta)^{2} \frac{1}{2 \varepsilon_{11}} \sum_{K} \alpha_{u}\left\|P_{u}^{\perp}\left(\nabla \cdot \boldsymbol{\sigma}_{h}\right)\right\|_{K}^{2}-2 \frac{\delta_{0} h}{2 \mu} \frac{1}{2 \varepsilon_{12}}(1-\beta)(2 \beta \mu)^{2} \frac{2 c_{\mathrm{tr}}}{h} \sum_{K}\left\|\nabla^{\mathrm{s}} \boldsymbol{u}_{h}\right\|_{K}^{2} \\
& +\sum_{K} \alpha_{\sigma} C_{\sigma}\left\|P_{\sigma}\left(-(1-\beta) \nabla^{\mathrm{s}} \boldsymbol{u}_{h}+\frac{\lambda}{2 \mu}\left(\boldsymbol{a}_{h} \cdot \nabla \boldsymbol{\sigma}_{h}-\dot{\boldsymbol{\sigma}}_{h}^{* *}\right)\right)\right\|_{K}^{2} \\
& +\sum_{K} \alpha_{\sigma}\left[1-2 \frac{1}{2 \varepsilon_{10}}-2\left(\frac{\alpha_{\sigma}}{2 \mu} \frac{\varepsilon_{9}}{2}+\alpha_{u} \frac{\alpha_{\sigma}}{h^{2}} c_{\mathrm{inv}}^{2} \frac{\varepsilon_{11}}{2}+2 c_{\mathrm{tr}} \delta_{0} \frac{\alpha_{\sigma}}{2 \mu} \frac{\varepsilon_{12}}{2}\right)\right. \\
& \left.\quad-2\left(\frac{\alpha_{\sigma}}{2 \mu}\right)^{2}\left(4\left(\frac{\lambda\left\|\boldsymbol{a}_{h}\right\|_{K}}{h}\right)^{2}+4\left(\lambda\left\|\nabla^{\mathrm{s}} \boldsymbol{a}_{h}\right\|_{K}\right)^{2}+2\left(\lambda\left\|\nabla^{\mathrm{as}} \boldsymbol{a}_{h}\right\|_{K}\right)^{2}\right) \frac{\varepsilon_{13}}{2}\right]\left\|P_{\sigma}\left(\frac{\lambda}{2 \mu} \dot{\boldsymbol{\sigma}}_{h}^{*}\right)\right\|_{K}^{2} \\
& -2 \frac{1}{2 \varepsilon_{13}} \sum_{K} \alpha_{\sigma}\left\|P_{\sigma}^{\perp}\left(-(1-\beta) \nabla^{\mathrm{s}} \boldsymbol{u}_{h}+\frac{\lambda}{2 \mu}\left(\boldsymbol{a}_{h} \cdot \nabla \boldsymbol{\sigma}_{h}-\dot{\boldsymbol{\sigma}}_{h}^{* *}\right)\right)\right\|_{K}^{2}-2 \frac{1}{2 \varepsilon_{13}} \sum_{K} \alpha_{\sigma}\left\|P_{\sigma}^{\perp}\left(\frac{\lambda}{2 \mu} \dot{\boldsymbol{\sigma}}_{h}^{*}\right)\right\|_{K}^{2}
\end{aligned}
$$

for any $\varepsilon_{i}, i=9,10,11,12,13$, with

$$
\begin{aligned}
C_{\sigma} & :=1-2 \frac{\varepsilon_{10}}{2}-2\left(\frac{\alpha_{\sigma}}{2 \mu} \frac{\varepsilon_{9}}{2}+\alpha_{u} \frac{\alpha_{\sigma}}{h^{2}} c_{\mathrm{inv}}^{2} \frac{\varepsilon_{11}}{2}+2 c_{\mathrm{tr}} \delta_{0} \frac{\alpha_{\sigma}}{2 \mu} \frac{\varepsilon_{12}}{2}\right) \\
& -2\left(\frac{\alpha_{\sigma}}{2 \mu}\right)^{2}\left(4\left(\frac{\lambda\left\|\boldsymbol{a}_{h}\right\|_{K}}{h}\right)^{2}+4\left(\lambda\left\|\nabla^{\mathrm{s}} \boldsymbol{a}_{h}\right\|_{K}\right)^{2}+2\left(\lambda\left\|\nabla^{\mathrm{as}} \boldsymbol{a}_{h}\right\|_{K}\right)^{2}\right) \frac{\varepsilon_{13}}{2}
\end{aligned}
$$

It can be checked that the constants $\varepsilon_{i}, i=1, \ldots, 13$, can be taken such that

$$
C_{u}>0, \quad C_{p}>0, \quad C_{\sigma}>0
$$

where $C_{u}, C_{p}$ and $C_{\sigma}$ are given by (28), (30) and (32), respectively.

Let $\boldsymbol{V}_{h}=\boldsymbol{U}_{h 1}+\theta_{1} \boldsymbol{V}_{h 1}+\theta_{2} \boldsymbol{V}_{h 2}+\theta_{3} \boldsymbol{V}_{h 3}$. It is trivially verified that the parameters $\theta_{i}$ can be chosen small enough so as to obtain

$$
\begin{aligned}
B_{\text {stab }}\left(\boldsymbol{U}_{h}, \boldsymbol{V}_{h}\right) & \geq 2 \beta(1-\beta) \mu \sum_{K} C_{1}\left\|\nabla^{\mathrm{s}} \boldsymbol{u}_{h}\right\|^{2}+\frac{1}{2 \mu} \sum_{K} C_{2}\left\|\boldsymbol{\sigma}_{h}\right\|^{2} \\
& +(1-\beta) \sum_{K} \alpha_{u} C_{3}\left\|P_{u, 0}\left(\rho \boldsymbol{a}_{h} \cdot \nabla \boldsymbol{u}_{h}+\nabla p_{h}-\nabla \cdot \boldsymbol{\sigma}_{h}\right)\right\|^{2} \\
& +(1-\beta) \sum_{K} \alpha_{u} C_{4}\left\|P_{u}^{\perp}\left(\rho \boldsymbol{a}_{h} \cdot \nabla \boldsymbol{u}_{h}\right)\right\|^{2}+(1-\beta) \sum_{K} \alpha_{u} C_{5}\left\|P_{u}^{\perp}\left(\nabla p_{h}\right)\right\|^{2} \\
& +(1-\beta) \sum_{K} \alpha_{u} C_{6}\left\|P_{u}^{\perp}\left(\nabla \cdot \boldsymbol{\sigma}_{h}\right)\right\|^{2}
\end{aligned}
$$




$$
\begin{aligned}
& +(1-\beta) \sum_{K} \alpha_{p} C_{7}\left\|P_{p}^{\perp}\left(\nabla \cdot \boldsymbol{u}_{h}\right)\right\|^{2}+(1-\beta) \sum_{K} \alpha_{p} C_{8}\left\|P_{p}\left(\nabla \cdot \boldsymbol{u}_{h}\right)\right\|^{2} \\
& +\sum_{K} \alpha_{\sigma} C_{9}\left\|P_{\sigma}\left(-(1-\beta) \nabla^{\mathrm{s}} \boldsymbol{u}_{h}+\frac{\lambda}{2 \mu}\left(\boldsymbol{a}_{h} \cdot \nabla \boldsymbol{\sigma}_{h}-\dot{\boldsymbol{\sigma}}_{h}^{* *}\right)\right)\right\|^{2} \\
& +\sum_{K} \alpha_{\sigma} C_{10}\left\|P_{\sigma}^{\perp}\left(-(1-\beta) \nabla^{\mathrm{s}} \boldsymbol{u}_{h}+\frac{\lambda}{2 \mu}\left(\boldsymbol{a}_{h} \cdot \nabla \boldsymbol{\sigma}_{h}-\dot{\boldsymbol{\sigma}}_{h}^{* *}\right)\right)\right\|^{2} \\
& +\frac{\delta_{0} h}{2 \mu} \sum_{E} C_{11}\left\|\llbracket \boldsymbol{n} p_{h}-\boldsymbol{n} \cdot \boldsymbol{\sigma}_{h} \rrbracket\right\|^{2}+\frac{1}{2 \mu} \sum_{K} \alpha_{\sigma} C_{12}\left\|\lambda P_{\sigma}\left(\dot{\boldsymbol{\sigma}}_{h}^{*}\right)\right\|^{2}
\end{aligned}
$$

with the various constants appearing in this expression given by

$$
\begin{aligned}
& C_{1}=1-\theta_{1} 2 \beta c_{\mathrm{inv}}^{2} \alpha_{u} \frac{\mu}{h^{2}}\left(\frac{1}{2 \varepsilon_{1}}+\frac{4 c_{\mathrm{tr}}}{2 \varepsilon_{2}}+\frac{2 c_{\mathrm{tr}} \delta_{0}}{2 \varepsilon_{5}}\right)-\theta_{2} \frac{\alpha_{p}}{\mu}\left(4 \delta_{0} \beta c_{\mathrm{tr}} \varepsilon_{8}\right)-\theta_{3} 2 \delta_{0} \beta c_{\mathrm{tr}} \frac{1}{2 \varepsilon_{12}} \\
& C_{2}=1-2 \lambda\left\|\nabla \boldsymbol{a}_{h}\right\|_{K}-4 \lambda \alpha_{\sigma} \frac{\lambda}{2 \mu}\left\|\nabla^{\mathrm{s}} \boldsymbol{a}_{h}\right\|_{K}^{2}-\theta_{3} \frac{1}{2 \varepsilon_{9}} \\
& -\left(\alpha_{\sigma} \frac{\lambda}{2 \mu}\left\|\nabla^{\mathrm{s}} \boldsymbol{a}_{h}\right\|_{K}\right)^{2}\left(\theta_{1}\left(2(1-\beta) \frac{2 \mu}{\alpha_{\sigma}} \frac{1}{2 \varepsilon_{6}}\right)+\theta_{3}\left(2 \frac{2 \mu}{\alpha_{\sigma}} \frac{1}{2 \varepsilon_{13}}\right)\right) \\
& C_{3}=\theta_{1}\left(1-\frac{\varepsilon_{1}}{2}-\frac{\varepsilon_{2}}{4} c_{\mathrm{tr}}-\left(\alpha_{u} \frac{\alpha_{p}}{h^{2}} \frac{\varepsilon_{3}}{2}+\alpha_{u} \frac{\rho\left\|\boldsymbol{a}_{h}\right\|_{K}}{h} \frac{\varepsilon_{4}}{2}+\alpha_{u} \frac{2 \mu}{h^{2}} \beta^{2} \delta_{0} c_{\mathrm{tr}} \frac{\varepsilon_{5}}{2}+\alpha_{u} \frac{\alpha_{\sigma}}{h^{2}} \frac{\varepsilon_{6}}{2}\right) c_{\mathrm{inv}}^{2}\right) \\
& C_{4}=1-\theta_{1} \alpha_{u} \frac{\mu}{h^{2}} \frac{\rho\left\|\boldsymbol{a}_{h}\right\|_{K} h}{\mu} \frac{1}{2 \varepsilon_{4}} \\
& C_{5}=1-\theta_{2} \frac{\varepsilon_{7}}{2} \\
& C_{6}=1-\theta_{3}(1-\beta) \frac{1}{2 \varepsilon_{11}} \\
& C_{7}=1-\theta_{1} \frac{1}{2 \varepsilon_{3}} \\
& C_{8}=\theta_{2}\left(1-c_{\mathrm{inv}}^{2} \alpha_{u} \frac{\alpha_{p}}{h^{2}} \frac{1}{2 \varepsilon_{7}}-\delta_{0} c_{\mathrm{tr}} \frac{1}{2 \varepsilon_{8}}\right) \\
& C_{9}=\theta_{3}\left(1-2 \frac{\varepsilon_{10}}{2}-2\left(\frac{\alpha_{\sigma}}{2 \mu} \frac{\varepsilon_{9}}{2}+\alpha_{u} \frac{\alpha_{\sigma}}{h^{2}} c_{\mathrm{inv}}^{2} \frac{\varepsilon_{11}}{2}+2 c_{\mathrm{tr}} \delta_{0} \frac{\alpha_{\sigma}}{2 \mu} \frac{\varepsilon_{12}}{2}\right)\right. \\
& \left.-2\left(\frac{\alpha_{\sigma}}{2 \mu}\right)^{2}\left(4\left(\frac{\lambda\left\|\boldsymbol{a}_{h}\right\|_{K}}{h}\right)^{2}+4\left(\lambda\left\|\nabla^{\mathrm{s}} \boldsymbol{a}_{h}\right\|_{K}\right)^{2}+2\left(\lambda\left\|\nabla^{\mathrm{as}} \boldsymbol{a}_{h}\right\|_{K}\right)^{2}\right) \frac{\varepsilon_{13}}{2}\right) \\
& C_{10}=1-\theta_{1} 2(1-\beta) \frac{1}{2 \varepsilon_{6}}-\theta_{3} 2 \frac{1}{2 \varepsilon_{13}} \\
& C_{11}=1-2 \theta_{1} 2(1-\beta)\left(\alpha_{u} \frac{\mu}{\delta_{0} h^{2}} \frac{1}{2 \varepsilon_{2}}+\frac{1}{2 \varepsilon_{5}}\right)-\theta_{2}(1-\beta) \frac{\alpha_{p}}{\mu} \varepsilon_{8}-\theta_{3} \frac{1}{2 \varepsilon_{12}} \\
& C_{12}=\theta_{3} \frac{\alpha_{\sigma}}{2 \mu}\left(1-2 \frac{1}{2 \varepsilon_{10}}-2\left(\frac{\alpha_{\sigma}}{2 \mu} \frac{\varepsilon_{9}}{2}+\alpha_{u} \frac{\alpha_{\sigma}}{h^{2}} c_{\mathrm{inv}}^{2} \frac{\varepsilon_{11}}{2}+2 c_{\mathrm{tr}} \delta_{0} \frac{\alpha_{\sigma}}{2 \mu} \frac{\varepsilon_{12}}{2}\right)\right. \\
& \left.-2\left(\frac{\alpha_{\sigma}}{2 \mu}\right)^{2}\left(4\left(\lambda \frac{\left\|\boldsymbol{a}_{h}\right\|_{K}}{h}\right)^{2}+4\left(\lambda\left\|\nabla^{\mathrm{s}} \boldsymbol{a}_{h}\right\|_{K}\right)^{2}+2\left(\lambda\left\|\nabla^{\mathrm{as}} \boldsymbol{a}_{h}\right\|_{K}\right)^{2}\right) \frac{\varepsilon_{13}}{2}\right)
\end{aligned}
$$


all positive for $\lambda$ (or the Weissenberg number) small enough and the constants $c_{i}, i=1,2,3,4,5$ in (18)-(20) large enough.

Comparing the terms in the right-hand-side of (33) and the definition (23), it is seen that the former bounds $\left\|P_{u, 0}\left(\rho \boldsymbol{a}_{h} \cdot \nabla \boldsymbol{u}_{h}+\nabla p_{h}-\nabla \cdot \boldsymbol{\sigma}_{h}\right)\right\|$ and $\left\|P^{\perp}\left(\rho \boldsymbol{a}_{h} \cdot \nabla \boldsymbol{u}_{h}+\nabla p_{h}-\nabla \cdot \boldsymbol{\sigma}_{h}\right)\right\|$. Assumption H2 allows us to guarantee that it also bounds $\left\|\rho \boldsymbol{a}_{h} \cdot \nabla \boldsymbol{u}_{h}+\nabla p_{h}-\nabla \cdot \boldsymbol{\sigma}_{h}\right\|$. Therefore, for each $\boldsymbol{U}_{h}$ we have found $\boldsymbol{V}_{h}$ such that

$$
B_{\text {stab }}\left(\boldsymbol{U}_{h}, \boldsymbol{V}_{h}\right) \geq C\left\|\boldsymbol{U}_{h}\right\|_{W}^{2}
$$

In fact, it is seen from (33) that we could have included the term $\frac{1}{2 \mu} C_{12}\left\|\lambda P_{\sigma}\left(\dot{\boldsymbol{\sigma}}_{h}^{*}\right)\right\|^{2}$ in the working norm, which gives control on the finite element part of $\boldsymbol{\sigma}_{h} \cdot \nabla^{\mathrm{s}} \boldsymbol{a}_{h}+\nabla^{\mathrm{s}} \boldsymbol{a}_{h} \cdot \boldsymbol{\sigma}_{h}$.

On the other hand, it is easily checked that $\left\|\boldsymbol{V}_{h}\right\|_{W} \leq C\left\|\boldsymbol{U}_{h}\right\|_{W}$. Using this fact we have shown that for each $\boldsymbol{U}_{h} \in \mathcal{X}_{h}$ there exist $\boldsymbol{V}_{h} \in \mathcal{X}_{h}$ such that $B_{\text {stab }}\left(\boldsymbol{U}_{h}, \boldsymbol{V}_{h}\right) \geq C\left\|\boldsymbol{U}_{h}\right\|_{W}\left\|\boldsymbol{V}_{h}\right\|_{W}$, from where the theorem follows.

Once stability is established, a more or less standard procedure leads to convergence. The objective is to show that the error function of the method is

$$
E(h):=\sqrt{\mu} \varepsilon_{1}(\boldsymbol{u})+\sqrt{\mu} \sum_{K} \sqrt{\frac{\rho\left\|\boldsymbol{a}_{h}\right\|_{K} h}{\mu}} \varepsilon_{1, K}(\boldsymbol{u})+\frac{1}{\sqrt{\mu}} \varepsilon_{0}(\boldsymbol{\sigma})+\frac{1}{\sqrt{\mu}} \sum_{K} \frac{\lambda\left\|\boldsymbol{a}_{h}\right\|_{K}}{h} \varepsilon_{0, K}(\boldsymbol{\sigma})+\frac{1}{\sqrt{\mu}} \varepsilon_{0}(p)
$$

This expression allows us to see how the error will deteriorate in terms of the local Reynolds number and the local Weissenberg number, defined as the factors that multiply $\varepsilon_{1, K}(\boldsymbol{u})$ and $\varepsilon_{0, K}(\boldsymbol{\sigma})$, respectively.

The first preliminary result we need concerns the consistency of the formulation:

Lemma 1 (Consistency). Let $\boldsymbol{U} \in \mathcal{X}$ be the solution of the continuous problem and $\boldsymbol{U}_{h} \in \mathcal{X}_{h}$ the finite element solution. If $\boldsymbol{f} \in \mathcal{V}_{h}$ and $\boldsymbol{U}$ is regular enough, so that $B_{\text {stab }}\left(\boldsymbol{U}, \boldsymbol{V}_{h}\right)$ is well defined, then

$$
B_{\text {stab }}\left(\boldsymbol{U}-\boldsymbol{U}_{h}, \boldsymbol{V}_{h}\right) \leq C E(h)\left\|\boldsymbol{V}_{h}\right\|_{W}
$$

for all $\boldsymbol{V}_{h} \in \boldsymbol{X}_{h}$, where $E(h)$ is given by (34).

Proof. Obviously, the Galerkin terms do not contribute to the consistency error. In the constitutive equation the stabilization term is residual based and the consistency is also satisfied by construction, the same as in the continuity equation. It only remains to show that $S_{1}^{\perp}$ has a consistency error bounded as (35) indicates. This easily follows from the fact that the orthogonal projection $P^{\perp}$ onto an appropriate finite element space (without boundary conditions) satisfies $\left\|P^{\perp}(v)\right\| \leq C \varepsilon_{0}(v)$ for any function $v$.

The second preliminary lemma provides an interpolation error in terms of the working norm $\|\cdot\|_{W}$ and the stabilized form $B_{\text {stab }}$ for the continuous solution $\boldsymbol{U}=[\boldsymbol{u}, p, \boldsymbol{\sigma}] \in \mathcal{X}$, assumed to have enough regularity:

Lemma 2 (Interpolation error). Let $\boldsymbol{U} \in \mathcal{X}$ be the solution of the continuous problem, assumed to be regular enough, and $\tilde{\boldsymbol{U}}_{h} \in \mathcal{X}_{h}$ its best finite element approximation. Then, for $\lambda$ small enough the following inequalities hold:

$$
B_{\text {stab }}\left(\boldsymbol{U}-\tilde{\boldsymbol{U}}_{h}, \boldsymbol{V}_{h}\right) \leq C E(h)\left\|\boldsymbol{V}_{h}\right\|_{W}
$$




$$
\left\|\boldsymbol{U}-\tilde{\boldsymbol{U}}_{h}\right\|_{W} \leq C E(h)
$$

Proof. Set $\boldsymbol{e}_{u}=\boldsymbol{u}-\tilde{\boldsymbol{u}}_{h}, e_{p}=p-\tilde{p}_{h}$ and $\boldsymbol{e}_{\sigma}=\boldsymbol{\sigma}-\tilde{\boldsymbol{\sigma}}_{h}$. The proof of (37) follows from a repeated application of Schwarz's inequality and then Young's inequality for scalars. Boundary terms can be treated using (21). One gets

$$
\begin{aligned}
\left\|\boldsymbol{U}-\tilde{\boldsymbol{U}}_{h}\right\|_{W}^{2} & \leq 2 \mu \sum_{K}\left(\beta+\beta \frac{\varepsilon_{1}}{2}+\frac{\alpha_{p}}{2 \mu}+\frac{\alpha_{\sigma}}{2 \mu} \beta^{2} \frac{1}{2 \varepsilon_{3}}+2 \frac{\alpha_{\sigma}}{2 \mu}\left(1+\frac{\varepsilon_{3}}{2}\right)+2 \delta_{0} c_{\mathrm{tr}} \beta^{2}\left(1+2 \beta \frac{\varepsilon_{4}}{2}\right)\right)\left\|\nabla^{\mathrm{s}} \boldsymbol{e}_{u}\right\|_{K}^{2} \\
& +2 \mu \sum_{K} \alpha_{u} \frac{\rho\left\|\boldsymbol{a}_{h}\right\|_{K}}{h} \frac{\rho \boldsymbol{a}_{h} \|_{K} h}{2 \mu}\left\|\nabla^{\mathrm{s}} \boldsymbol{e}_{u}\right\|_{K}^{2} \\
& +\frac{1}{2 \mu} \sum_{K}\left(1+\beta \frac{1}{2 \varepsilon_{1}}+\frac{1}{2 \varepsilon_{2}}+4 \lambda^{2}\left\|\nabla \boldsymbol{a}_{h}\right\|_{K}^{2} \frac{\varepsilon_{2}}{2}+(1-\beta) \alpha_{u} \frac{2 \mu}{h^{2}}+2 c_{\mathrm{tr}} \delta_{0}(2+\beta)\right)\left\|\boldsymbol{e}_{\sigma}\right\|_{K}^{2} \\
& +\frac{1}{2 \mu} \sum_{K} \frac{\alpha_{\sigma}}{2 \mu}\left(2\left(\frac{\lambda\left\|\boldsymbol{a}_{h}\right\|_{K}}{h}-\lambda\left\|\nabla^{\mathrm{as}} \boldsymbol{a}_{h}\right\|_{K}\right)^{2}\left(1+2 \frac{\varepsilon_{3}}{2}\right)+\lambda\left\|\nabla^{\mathrm{s}} \boldsymbol{a}_{h}\right\|_{K}^{2}\left(\frac{1}{2}-2 \frac{\varepsilon_{3}}{2}\right)\right)\left\|\boldsymbol{e}_{\sigma}\right\|_{K}^{2} \\
& +\frac{1}{2 \mu} \sum_{K}\left(\alpha_{u} \frac{2 \mu}{h^{2}}+2 c_{\mathrm{tr}} \delta_{0}\left(1+2 \beta \frac{\varepsilon_{4}}{2}\right)\right)\left\|e_{p}\right\|_{K}^{2}
\end{aligned}
$$

for any constants $\varepsilon_{i}, i=1,2,3$ (not to be confused with the interpolation errors). Estimate (37) follows from the definition of the error function in (34) and assuming $\lambda$ to be small enough (this assumption allows us not to include the gradient of $\boldsymbol{a}_{h}$ in the error function).

To prove (36) one needs to apply repeatedly Young's inequality and use the expression of the stabilization parameters, as well as classical interpolation estimates. To treat the boundary terms, note that according to (21) we may write

$$
\sum_{K}\left\|v-\tilde{v}_{h}\right\|_{\partial K}^{2} \leq C\left(h^{-1} \varepsilon_{0}^{2}(v)+h \varepsilon_{1}^{2}(v)\right)=C h^{-1} \varepsilon_{0}^{2}(v)
$$

for both continuous and discontinuous functions $v$.

The bound one finally obtains is

$$
\begin{aligned}
B_{\text {stab }} & \left(\boldsymbol{U}-\tilde{\boldsymbol{U}}_{h}, \boldsymbol{V}_{h}\right) \\
\leq & \sqrt{\mu} \varepsilon_{1}(\boldsymbol{u})\left(\sum_{K}\left(2 \beta \sqrt{\mu}\left\|\nabla^{\mathrm{s}} \boldsymbol{v}_{h}\right\|_{K}+\frac{h}{\sqrt{\mu}}\left\|-\nabla q_{h}+(1-\beta) \nabla \cdot \boldsymbol{\tau}_{h}\right\|_{K}+\frac{\rho\left\|\boldsymbol{a}_{h}\right\| h}{\mu} \frac{\sqrt{\mu}}{c_{\mathrm{inv}}}\left\|\nabla \boldsymbol{v}_{h}\right\|_{K}\right)\right. \\
& \left.+\sum_{E} \sqrt{c_{\mathrm{tr}}} \frac{\sqrt{h}}{\sqrt{\mu}}\left\|\llbracket \boldsymbol{n} q_{h}-(1-\beta) \boldsymbol{n} \cdot \boldsymbol{\tau}_{h} \rrbracket\right\|_{E}\right) \\
+ & \frac{1}{\sqrt{\mu}} \varepsilon_{0}(\boldsymbol{\sigma}) \sum_{K}\left(\sqrt{\mu}\left\|\nabla^{\mathrm{s}} \boldsymbol{v}_{h}\right\|_{K}+\frac{1}{2 \sqrt{\mu}}\left\|\boldsymbol{\tau}_{h}\right\|_{K}+\frac{\lambda\left\|\boldsymbol{a}_{h}\right\|_{K}}{h} c_{\mathrm{inv}} \frac{1}{2 \sqrt{\mu}}\left\|\boldsymbol{\tau}_{h}\right\|_{K}+\lambda\left\|\nabla \boldsymbol{a}_{h}\right\|_{K} \frac{\lambda}{\sqrt{\mu}}\left\|\boldsymbol{\tau}_{h}\right\|_{K}\right) \\
+ & (1-\beta) \frac{c_{\mathrm{inv}}}{\sqrt{\mu}} \varepsilon_{0}(\boldsymbol{\sigma}) \sum_{K} \alpha_{u} \frac{\mu}{h^{2}} \frac{c_{\mathrm{inv}}}{\sqrt{\mu}}\left\|\boldsymbol{\tau}_{h}\right\| \\
+ & \sqrt{\mu} \varepsilon_{1}(\boldsymbol{u}) \sum_{K}\left(\frac{\alpha_{p}}{\mu} \sqrt{\mu}\left\|\nabla \cdot \boldsymbol{v}_{h}\right\|_{K}+\alpha_{u} \frac{\mu}{h^{2}}\left(\frac{\rho\left\|\boldsymbol{a}_{h}\right\|_{K} h}{\mu}\right)^{2} \sqrt{\mu}\left\|\nabla \boldsymbol{v}_{h}\right\|_{K}+(1-\beta) \frac{\alpha_{\sigma}}{\mu} \sqrt{\mu}\left\|\nabla^{\mathrm{s}} \boldsymbol{v}_{h}\right\|_{K}\right)
\end{aligned}
$$




$$
\begin{aligned}
& +\frac{1}{\sqrt{\mu}} \varepsilon_{0}(p)\left(\sqrt{\mu}\left\|\nabla \cdot \boldsymbol{v}_{h}\right\|\right)+\frac{1}{\sqrt{\mu}} \varepsilon_{0}(p) \sum_{K}\left(\alpha_{u} \frac{\mu}{h^{2}} \frac{c_{\mathrm{inv}}^{2}}{\sqrt{\mu}}\left\|q_{h}\right\|_{K}\right) \\
& +\frac{\delta_{0}}{2} \frac{1}{\sqrt{\mu}}\left(\varepsilon_{0}(p)+\varepsilon_{0}(\boldsymbol{\sigma})+2 \beta \mu \varepsilon_{1}(\boldsymbol{u})\right) \sum_{E} \frac{\sqrt{h}}{\sqrt{\mu}}\left\|\llbracket \boldsymbol{n} q_{h}-(1-\beta) \boldsymbol{n} \cdot \boldsymbol{\tau}_{h}+2 \beta \mu \boldsymbol{n} \cdot \nabla^{\mathrm{s}} \boldsymbol{v}_{h} \rrbracket\right\|_{E} \\
& +(1-\beta) \sqrt{\mu} \varepsilon_{1}(\boldsymbol{u}) \sum_{K} \frac{\alpha_{\sigma}}{\mu} \frac{1}{2 \sqrt{\mu}}\left\|\lambda\left(\boldsymbol{a}_{h} \cdot \nabla \boldsymbol{\tau}_{h}+\boldsymbol{\tau}_{h} \cdot\left(\nabla \boldsymbol{a}_{h}\right)^{T}+\nabla \boldsymbol{a}_{h} \cdot \boldsymbol{\tau}_{h}\right)\right\| \\
& +\frac{1}{2 \sqrt{\mu}} \varepsilon_{0}(\boldsymbol{\sigma}) \sum_{K} \sqrt{\mu} \frac{\alpha_{\sigma}}{\mu}\left(\left\|\nabla^{\mathrm{s}} \boldsymbol{v}_{h}\right\|_{K}+\left\|\frac{\lambda}{2 \mu}\left(\boldsymbol{a}_{h} \cdot \nabla \boldsymbol{\tau}_{h}+\boldsymbol{\tau}_{h} \cdot\left(\nabla \boldsymbol{a}_{h}\right)^{T}+\nabla \boldsymbol{a}_{h} \cdot \boldsymbol{\tau}_{h}\right)\right\|_{K}\right) \\
& \\
& \left(\frac{\lambda\left\|\boldsymbol{a}_{h}\right\|_{K}}{h} c_{\mathrm{inv}}+2 \lambda\left\|\nabla \boldsymbol{a}_{h}\right\|_{K}\right)
\end{aligned}
$$

All the terms have been organized to see that they are bounded by $C E(h)\left\|\boldsymbol{V}_{h}\right\|_{W}$, from where (36) follows.

We are finally in a position to prove convergence. The proof is standard, but we include it for completeness.

Theorem 2 (Convergence). Let $\boldsymbol{U}=[\boldsymbol{u}, p, \boldsymbol{\sigma}] \in \mathcal{X}$ be the solution of the continuous problem, and suppose that the assumptions of Theorem 1 hold. Then there exist a constant $C>0$ such that

$$
\left\|\boldsymbol{U}-\boldsymbol{U}_{h}\right\|_{W} \leq C E(h)
$$

Proof. Consider the finite element function $\tilde{\boldsymbol{U}}_{h}-\boldsymbol{U}_{h} \in \mathcal{X}_{h}$, where as in Lemma $2, \tilde{\boldsymbol{U}}_{h} \in \mathcal{X}_{h}$ is the best finite element approximation to $\boldsymbol{U}$. Starting from the inf-sup condition, it follows that there exist $\boldsymbol{V}_{h} \in \mathcal{X}_{h}$ such that

$$
\begin{aligned}
C\left\|\tilde{\boldsymbol{U}}_{h}-\boldsymbol{U}_{h}\right\|_{W}\left\|\boldsymbol{V}_{h}\right\|_{W} & \leq B_{\text {stab }}\left(\tilde{\boldsymbol{U}}_{h}-\boldsymbol{U}_{h}, \boldsymbol{V}_{h}\right) \\
& =B_{\text {stab }}\left(\tilde{\boldsymbol{U}}_{h}-\boldsymbol{U}, \boldsymbol{V}_{h}\right)+B_{\text {stab }}\left(\boldsymbol{U}-\boldsymbol{U}_{h}, \boldsymbol{V}_{h}\right) \\
& \leq C E(h)\left\|\boldsymbol{V}_{h}\right\|_{W}
\end{aligned}
$$

using Lemma 1 and (36) in Lemma 2, from where

$$
\left\|\tilde{\boldsymbol{U}}_{h}-\boldsymbol{U}_{h}\right\|_{W} \leq C E(h)
$$

The theorem follows using this bound in the triangle inequality

$$
\left\|\boldsymbol{U}-\boldsymbol{U}_{h}\right\|_{W} \leq\left\|\boldsymbol{U}-\tilde{\boldsymbol{U}}_{h}\right\|_{W}+\left\|\tilde{\boldsymbol{U}}_{h}-\boldsymbol{U}_{h}\right\|_{W}
$$

and (37) in Lemma 2.

Clearly, this convergence result is optimal.

\subsection{Stability and convergence in natural norms}

The next step will be to prove stability and convergence in a natural norm, that is yo say, in the norm of the space where the continuous problem is posed, and not in the mesh dependent norm (23). Even though 
the results to be presented are the expected ones, the analysis presented up to this point has highlighted the role played by the stabilization terms of the formulation. Obviously, since the natural norm does not include any control on the convective terms, stability and convergence in this norm is only meaningful in the case of small cell Reynolds numbers and small cell Weissenberg numbers. This is the situation considered in the following.

Theorem 3 (Stability and convergence in natural norms). Suppose that the assumptions of Theorem 1 hold and the cell Reynolds numbers and cell Weissenberg numbers are small. Then, the solution of the discrete problem $\boldsymbol{U}_{h}=\left[\boldsymbol{u}_{h}, p_{h}, \boldsymbol{\sigma}_{h}\right] \in \mathcal{X}_{h}$ can be bounded as

$$
\sqrt{\mu}\left\|\boldsymbol{u}_{h}\right\|_{H^{1}(\Omega)}+\frac{1}{\sqrt{\mu}}\left\|\boldsymbol{\sigma}_{h}\right\|+\frac{1}{\sqrt{\mu}}\left\|p_{h}\right\| \leq \frac{C}{\sqrt{\mu}}\|\boldsymbol{f}\|_{H^{-1}(\Omega)}
$$

Moreover, if the solution of the continuous problem $\boldsymbol{U}=[\boldsymbol{u}, p, \boldsymbol{\sigma}] \in \mathcal{X}$ is regular enough, the following error estimate holds:

$$
\sqrt{\mu}\left\|\boldsymbol{u}-\boldsymbol{u}_{h}\right\|_{H^{1}(\Omega)}+\frac{1}{\sqrt{\mu}}\left\|\boldsymbol{\sigma}-\boldsymbol{\sigma}_{h}\right\|+\frac{1}{\sqrt{\mu}}\left\|p-p_{h}\right\| \leq C E(h)
$$

Proof. It is clear that

$$
\left\langle\boldsymbol{f}, \boldsymbol{v}_{h}\right\rangle \leq \frac{C}{\sqrt{\mu}}\|\boldsymbol{f}\|_{H^{-1}(\Omega)} \sqrt{\mu}\left\|\boldsymbol{v}_{h}\right\|_{H^{1}(\Omega)} \leq \frac{C}{\sqrt{\mu}}\|\boldsymbol{f}\|_{H^{-1}(\Omega)}\left\|\boldsymbol{V}_{h}\right\|_{W}
$$

where $\boldsymbol{V}_{h}=\left[\boldsymbol{v}_{h}, q_{h}, \boldsymbol{\tau}_{h}\right] \in \mathcal{X}_{h}$ is arbitrary. Therefore the inf-sup condition proved in Theorem 1 implies that:

$$
\left\|\boldsymbol{U}_{h}\right\|_{W} \leq \frac{C}{\sqrt{\mu}}\|\boldsymbol{f}\|_{H^{-1}(\Omega)}
$$

that is to say,

$$
\begin{aligned}
& 2 \beta \mu(1-\beta)\left\|\nabla^{\mathrm{s}} \boldsymbol{u}_{h}\right\|^{2}+\frac{1}{2 \mu}\left\|\boldsymbol{\sigma}_{h}\right\|^{2}+\sum_{K} \alpha_{p}\left\|\nabla \cdot \boldsymbol{u}_{h}\right\|_{K}^{2}+\sum_{K} \alpha_{u}\left\|\rho \boldsymbol{a}_{h} \cdot \nabla \boldsymbol{u}_{h}+\nabla p_{h}-\nabla \cdot \boldsymbol{\sigma}_{h}\right\|_{K}^{2} \\
& +(1-\beta) \sum_{K} \alpha_{u}\left\|P_{u}^{\perp}\left(\nabla \cdot \boldsymbol{\sigma}_{h}\right)\right\|_{K}^{2}+\sum_{K} \alpha_{u}\left\|P_{u}^{\perp}\left(\nabla p_{h}\right)\right\|_{K}^{2}+\sum_{K} \alpha_{u}\left\|P_{u}^{\perp}\left(\rho \boldsymbol{a}_{h} \cdot \nabla \boldsymbol{u}_{h}\right)\right\|_{K}^{2} \\
& +\frac{\delta_{0} h}{2 \mu}(1-\beta) \sum_{E}\left\|\llbracket \boldsymbol{n} p_{h}-\boldsymbol{n} \cdot \boldsymbol{\sigma}_{h} \rrbracket\right\|_{E}^{2}+\sum_{K} \alpha_{\sigma}\left\|-(1-\beta) \nabla^{\mathrm{s}} \boldsymbol{u}_{h}+\frac{\lambda}{2 \mu}\left(\boldsymbol{a}_{h} \cdot \nabla \boldsymbol{\sigma}_{h}-\dot{\boldsymbol{\sigma}}_{h}^{* *}\right)\right\|_{K}^{2} \\
& \leq \frac{C}{\sqrt{\mu}}\|\boldsymbol{f}\|_{H^{-1}(\Omega)}
\end{aligned}
$$

Using an inverse inequality we can write:

$$
\sum_{K} \alpha_{u}\left\|\rho \boldsymbol{a}_{h} \cdot \nabla \boldsymbol{u}_{h}+\nabla p_{h}\right\|_{K}^{2} \leq \sum_{K} \alpha_{u}\left\|\left(\rho \boldsymbol{a}_{h} \cdot \nabla \boldsymbol{u}_{h}+\nabla p_{h}\right)-\nabla \cdot \boldsymbol{\sigma}_{h}\right\|_{K}^{2}+\sum_{K} \alpha_{u} \frac{\mu}{h^{2}} \frac{C}{\mu}\left\|\boldsymbol{\sigma}_{h}\right\|_{K}^{2}
$$

In this expression we only have control on $\rho \boldsymbol{a}_{h} \cdot \nabla \boldsymbol{u}_{h}+\nabla p_{h}$. However, we do not have explicit bounds over these two terms (and their errors) separately. Nevertheless, there is the possibility of bounding the pressure 
gradient making use of the control over the viscous term (viscous dominate case), since

$$
\sum_{K} \alpha_{u}\left\|\nabla p_{h}\right\|_{K}^{2} \leq \sum_{K} \alpha_{u}\left\|\rho \boldsymbol{a}_{h} \cdot \nabla \boldsymbol{u}_{h}+\nabla p_{h}\right\|_{K}^{2}+\sum_{K} \alpha_{u} \frac{\mu^{2}}{h^{2}}\left(\frac{\rho\left\|\boldsymbol{a}_{h}\right\|_{K} h}{\mu}\right)^{2}\left\|\nabla \boldsymbol{u}_{h}\right\|_{K}^{2}
$$

Note that this expression explodes with the cell Reynolds number $\operatorname{Re}_{h}:=\rho\left\|\boldsymbol{a}_{h}\right\|_{K} h \mu^{-1}$. We assume hereafter that this is bounded.

Using the trace inequality we can write

$$
(1-\beta) \frac{\delta_{0} h}{2 \mu} \sum_{E}\left\|\llbracket \boldsymbol{n} p_{h} \rrbracket\right\|_{E}^{2} \leq(1-\beta) \frac{\delta_{0} h}{2 \mu} \sum_{E}\left\|\llbracket \boldsymbol{n} p_{h}-\boldsymbol{n} \cdot \boldsymbol{\sigma}_{h} \rrbracket\right\|_{E}^{2}+(1-\beta) \frac{\delta_{0}}{2 \mu} 2 c_{\mathrm{tr}}\left\|\boldsymbol{\sigma}_{h}\right\|^{2}
$$

Thus, from (38), using Korn's inequality, the expression of $\alpha_{u}$ and noting that $0<\beta<1$, we have

$$
\mu\left\|\boldsymbol{u}_{h}\right\|_{H^{1}(\Omega)}^{2}+\frac{1}{\mu}\left\|\boldsymbol{\sigma}_{h}\right\|^{2}+\frac{h^{2}}{\mu} \sum_{K}\left\|\nabla p_{h}\right\|_{K}^{2}+\frac{h}{\mu} \sum_{E}\left\|\left[\boldsymbol{n} p_{h}\right]\right\|_{E}^{2} \lesssim \frac{1}{\mu}\|\boldsymbol{f}\|_{H^{-1}(\Omega)}^{2}
$$

To prove the $L^{2}$-stability for the pressure we rely on the inf-sup condition between the velocity and pressure spaces that holds for the continuous problem, as in [18]. Details can be found in this reference. This concludes the proof of the first part of the Theorem.

Let us proceed to prove convergence in natural norms. Theorem 2 implies that

$$
\begin{aligned}
& \mu\left\|\boldsymbol{u}-\boldsymbol{u}_{h}\right\|_{H^{1}(\Omega)}^{2}+\frac{1}{\mu}\left\|\boldsymbol{\sigma}-\boldsymbol{\sigma}_{h}\right\|^{2}+\sum_{K} \alpha_{u}\left\|\rho \boldsymbol{a}_{h} \cdot \nabla\left(\boldsymbol{u}-\boldsymbol{u}_{h}\right)+\nabla\left(p-p_{h}\right)-\nabla \cdot\left(\boldsymbol{\sigma}-\boldsymbol{\sigma}_{h}\right)\right\|_{K}^{2} \\
& +\frac{h}{\mu} \sum_{E}\left\|\llbracket \boldsymbol{n}\left(p-p_{h}\right)-\boldsymbol{n} \cdot\left(\boldsymbol{\sigma}-\boldsymbol{\sigma}_{h}\right) \rrbracket\right\|_{E}^{2}+\frac{h}{\mu} \sum_{E}\left\|\llbracket \mu \boldsymbol{n} \cdot \nabla^{\mathrm{s}}\left(\boldsymbol{u}-\boldsymbol{u}_{h}\right) \rrbracket\right\|_{E}^{2} \lesssim E^{2}(h)
\end{aligned}
$$

We may use now the same procedure as for proving stability. Assuming the cell Reynolds number to be small, using (21) and classical inverse estimates, we obtain

$$
\begin{aligned}
\frac{h^{2}}{\mu} \sum_{K}\left\|\nabla\left(p-p_{h}\right)\right\|_{K}^{2} & \lesssim \sum_{K} \alpha_{u}\left\|\rho \boldsymbol{a}_{h} \cdot \nabla\left(\boldsymbol{u}-\boldsymbol{u}_{h}\right)+\nabla\left(p-p_{h}\right)-\nabla \cdot\left(\boldsymbol{\sigma}-\boldsymbol{\sigma}_{h}\right)\right\|_{K}^{2} \\
& +\mu\left\|\boldsymbol{u}-\boldsymbol{u}_{h}\right\|_{H^{1}(\Omega)}^{2}+\frac{1}{\mu}\left\|\boldsymbol{\sigma}-\boldsymbol{\sigma}_{h}\right\|^{2} \\
\frac{h}{\mu} \sum_{E}\left\|\llbracket \boldsymbol{n}\left(p-p_{h}\right) \rrbracket\right\|_{E}^{2} & \lesssim \frac{h}{\mu} \sum_{E}\left\|\llbracket \boldsymbol{n}\left(p-p_{h}\right)-\boldsymbol{n} \cdot\left(\boldsymbol{\sigma}-\boldsymbol{\sigma}_{h}\right) \rrbracket\right\|_{E}^{2}+\frac{1}{\mu}\left\|\boldsymbol{\sigma}-\boldsymbol{\sigma}_{h}\right\|^{2}
\end{aligned}
$$

and therefore we have that

$$
\mu\left\|\boldsymbol{u}-\boldsymbol{u}_{h}\right\|_{H^{1}(\Omega)}^{2}+\frac{1}{\mu}\left\|\boldsymbol{\sigma}-\boldsymbol{\sigma}_{h}\right\|^{2}+\frac{h^{2}}{\mu} \sum_{K}\left\|\nabla\left(p-p_{h}\right)\right\|_{K}^{2}+\frac{h}{\mu} \sum_{E}\left\|\llbracket \boldsymbol{n}\left(p-p_{h}\right) \rrbracket\right\|_{E}^{2} \lesssim E^{2}(h)
$$

The error estimate in Theorem 3 can now be obtained using the same procedure as in [18], which relies once again on the inf-sup condition between the velocity and the pressure spaces that holds at the continuous level. 
To complete the analysis of the problem, let us obtain an $L^{2}$-error estimate for the velocity field. As usual, this can be proved using a duality argument and an elliptic regularity condition assuming the forcing term to be in $L^{2}(\Omega)$. The result we have is the following:

Theorem 4 ( $L^{2}$-error estimate for the velocity). Assume that the hypothesis of Theorem 3 hold and suppose that the continuous problem satisfies the elliptic regularity condition

$$
\sqrt{\mu}\|\boldsymbol{u}\|_{H^{2}(\Omega)}+\frac{1}{\sqrt{\mu}}\|\boldsymbol{\sigma}\|_{H^{1}(\Omega)}+\frac{1}{\sqrt{\mu}}\|p\|_{H^{1}(\Omega)} \leq \frac{C}{\sqrt{\mu}}\|\boldsymbol{f}\|
$$

Then

$$
\sqrt{\mu}\left\|\boldsymbol{u}-\boldsymbol{u}_{h}\right\| \lesssim h\left(\sqrt{\mu}\left\|\boldsymbol{u}-\boldsymbol{u}_{h}\right\|_{H^{1}(\Omega)}+\frac{1}{\sqrt{\mu}}\left\|\boldsymbol{\sigma}-\boldsymbol{\sigma}_{h}\right\|+\frac{1}{\sqrt{\mu}}\left\|p-p_{h}\right\|\right)
$$

Proof. The key point is to set the appropriate adjoint problem in our case. Let $[\boldsymbol{w}, \pi, \boldsymbol{S}] \in \mathcal{X}$ be the solution of the following problem:

$$
\begin{aligned}
-\beta \mu \Delta \boldsymbol{w}-\rho \boldsymbol{a} \cdot \nabla \boldsymbol{w}+(1-\beta) \nabla \cdot \boldsymbol{S}-\nabla \pi & =\frac{\mu}{l^{2}}\left(\boldsymbol{u}-\boldsymbol{u}_{h}\right) \\
-\nabla \cdot \boldsymbol{w} & =0 \\
\frac{1}{2 \mu} \boldsymbol{S}+\nabla^{\mathrm{s}} \boldsymbol{w}-\frac{\lambda}{2 \mu}\left(\boldsymbol{a} \cdot \nabla \boldsymbol{S}+\boldsymbol{S} \cdot(\nabla \boldsymbol{a})^{T}+\nabla \boldsymbol{a} \cdot \boldsymbol{S}\right) & =\mathbf{0}
\end{aligned}
$$

with $\boldsymbol{w}=\mathbf{0}$ on $\partial \Omega$ and where $l$ is a characteristic length scale of the problem that has been introduced to keep the dimensionality, but that will play no role in the final result. Let also $\left[\tilde{\boldsymbol{w}}_{h}, \tilde{\pi}_{h}, \tilde{\boldsymbol{S}}_{h}\right]$ be the best approximation to $[\boldsymbol{w}, \pi, \boldsymbol{S}]$ in $\boldsymbol{\mathcal { X }}_{h}$. Testing with $\left[\boldsymbol{u}-\boldsymbol{u}_{h}, p-p_{h}, \boldsymbol{\sigma}-\boldsymbol{\sigma}_{h}\right],\left[\boldsymbol{u}_{h}, p_{h}, \boldsymbol{\sigma}_{h}\right]$ being the solution of the problem, we obtain

$$
\begin{aligned}
\frac{\mu}{l^{2}} & \left\|\boldsymbol{u}-\boldsymbol{u}_{h}\right\|^{2}=B\left(\left[\boldsymbol{u}-\boldsymbol{u}_{h}, p-p_{h}, \boldsymbol{\sigma}-\boldsymbol{\sigma}_{h}\right],[\boldsymbol{w}, \pi, \boldsymbol{S}]\right) \\
& =B_{\mathrm{stab}}\left(\left[\boldsymbol{u}-\boldsymbol{u}_{h}, p-p_{h}, \boldsymbol{\sigma}-\boldsymbol{\sigma}_{h}\right],[\boldsymbol{w}, \pi, \boldsymbol{S}]\right) \\
& -\sum_{K} \alpha_{\sigma}\left\langle P_{\sigma}^{\perp}\left(\frac{1}{2 \mu} \boldsymbol{S}+\nabla^{\mathrm{s}} \boldsymbol{w}-\frac{\lambda}{2 \mu}\left(\boldsymbol{a} \cdot \nabla \boldsymbol{S}+\boldsymbol{S} \cdot(\nabla \boldsymbol{a})^{T}+\nabla \boldsymbol{a} \cdot \boldsymbol{S}\right)\right),\right. \\
& \left.P_{\sigma}^{\perp}\left(\nabla^{\mathrm{s}}\left(\boldsymbol{u}-\boldsymbol{u}_{h}\right)-\frac{\lambda}{2 \mu}\left(\boldsymbol{a}_{h} \cdot \nabla\left(\boldsymbol{\sigma}-\boldsymbol{\sigma}_{h}\right)+\left(\boldsymbol{\sigma}-\boldsymbol{\sigma}_{h}\right) \cdot\left(\nabla \boldsymbol{a}_{h}\right)^{T}+\nabla \boldsymbol{a}_{h} \cdot\left(\boldsymbol{\sigma}-\boldsymbol{\sigma}_{h}\right)\right)\right)\right\rangle_{K} \\
& -\sum_{K} \alpha_{p}\left(P_{p}^{\perp}(\nabla \cdot \boldsymbol{w}), P_{p}^{\perp}\left(\nabla \cdot\left(\boldsymbol{u}-\boldsymbol{u}_{h}\right)\right)\right)_{K} \\
& -\sum_{K} \alpha_{u}\left\langle P_{u}^{\perp}(\nabla \pi), P_{u}^{\perp}\left(\nabla\left(p-p_{h}\right)\right)\right\rangle_{K}-\sum_{K} \alpha_{u}\left\langle P_{u}^{\perp}\left(\rho \boldsymbol{a}_{h} \cdot \nabla \boldsymbol{w}\right), P_{u}^{\perp}\left(\rho \boldsymbol{a}_{h} \cdot \nabla\left(\boldsymbol{u}-\boldsymbol{u}_{h}\right)\right)\right\rangle_{K} \\
& -\sum_{K} \alpha_{u}\left\langle P_{u}^{\perp}(\nabla \cdot \boldsymbol{S}), P_{u}^{\perp}\left((1-\beta) \nabla \cdot\left(\boldsymbol{\sigma}-\boldsymbol{\sigma}_{h}\right)\right)\right\rangle_{K} \\
& -\frac{\delta_{0} h}{2 \mu} \sum_{E}\left\langle\llbracket \boldsymbol{n}\left(p-p_{h}\right)-(1-\beta) \boldsymbol{n} \cdot\left(\boldsymbol{\sigma}-\boldsymbol{\sigma}_{h}\right)+2 \beta \mu \boldsymbol{n} \cdot \nabla^{\mathrm{s}}\left(\boldsymbol{u}-\boldsymbol{u}_{h}\right) \rrbracket, \llbracket \boldsymbol{n} \pi-\boldsymbol{n} \cdot\left(\boldsymbol{S}+2 \beta \mu \nabla^{\mathrm{s}} \boldsymbol{w}\right) \rrbracket\right\rangle_{E}
\end{aligned}
$$

The second and the third row terms are zero because of the definition of the problem. The last term is zero because of the weak continuity of the stresses associated to the problem. Therefore only the first and 
the fourth terms need to be bounded. We have that

$$
\begin{aligned}
& \frac{\mu}{l^{2}}\left\|\boldsymbol{u}-\boldsymbol{u}_{h}\right\|^{2}=B_{\text {stab }}\left(\left[\boldsymbol{u}-\boldsymbol{u}_{h}, p-p_{h}, \boldsymbol{\sigma}-\boldsymbol{\sigma}_{h}\right],[\boldsymbol{w}, \pi, \boldsymbol{S}]\right) \\
& \quad-\sum_{K} \alpha_{u}\left\langle P_{u}^{\perp}(\nabla \pi), P_{u}^{\perp}\left(\nabla\left(p-p_{h}\right)\right)\right\rangle_{K}-\sum_{K} \alpha_{u}\left\langle P_{u}^{\perp}\left(\rho \boldsymbol{a}_{h} \cdot \nabla \boldsymbol{w}\right), P_{u}^{\perp}\left(\rho \boldsymbol{a}_{h} \cdot \nabla\left(\boldsymbol{u}-\boldsymbol{u}_{h}\right)\right)\right\rangle_{K} \\
& \quad-\sum_{K} \alpha_{u}\left\langle P_{u}^{\perp}(\nabla \cdot \boldsymbol{S}), P_{u}^{\perp}\left((1-\beta) \nabla \cdot\left(\boldsymbol{\sigma}-\boldsymbol{\sigma}_{h}\right)\right)\right\rangle_{K}
\end{aligned}
$$

Using the interpolation properties and the shift assumption it follows that

$$
\begin{aligned}
& \left\|\boldsymbol{w}-\tilde{\boldsymbol{w}}_{h}\right\|_{H^{1}(\Omega)} \lesssim h\|\boldsymbol{w}\|_{H^{2}(\Omega)} \lesssim h \frac{1}{l^{2}}\left\|\boldsymbol{u}-\boldsymbol{u}_{h}\right\| \\
& \left\|\boldsymbol{S}-\tilde{\boldsymbol{S}}_{h}\right\| \lesssim h\|\boldsymbol{S}\|_{H^{1}(\Omega)} \lesssim h \frac{\mu}{l^{2}}\left\|\boldsymbol{u}-\boldsymbol{u}_{h}\right\| \\
& \left\|\pi-\tilde{\pi}_{h}\right\| \lesssim h\|\pi\|_{H^{1}(\Omega)} \lesssim h \frac{\mu}{l^{2}}\left\|\boldsymbol{u}-\boldsymbol{u}_{h}\right\|
\end{aligned}
$$

From these expressions we obtain

$$
\begin{aligned}
& B_{\text {stab }}\left(\left[\boldsymbol{u}-\boldsymbol{u}_{h}, p-p_{h}, \boldsymbol{\sigma}-\boldsymbol{\sigma}_{h}\right],[\boldsymbol{w}, \pi, \boldsymbol{S}]\right) \\
& \quad=B_{\text {stab }}\left(\left[\boldsymbol{u}-\boldsymbol{u}_{h}, p-p_{h}, \boldsymbol{\sigma}-\boldsymbol{\sigma}_{h}\right],\left[\boldsymbol{w}-\tilde{\boldsymbol{w}}_{h}, \pi-\tilde{\pi}_{h}, \boldsymbol{S}-\tilde{\boldsymbol{S}}_{h}\right]\right) \\
& -\sum_{K} \alpha_{u}\left\langle P_{u}^{\perp}\left(\nabla\left(\pi-\tilde{\pi}_{h}\right)\right), P_{u}^{\perp}\left(\nabla\left(p-p_{h}\right)\right)\right\rangle_{K} \\
& -\sum_{K} \alpha_{u}\left\langle P_{u}^{\perp}\left(\rho \boldsymbol{a}_{h} \cdot \nabla\left(\boldsymbol{w}-\tilde{\boldsymbol{w}}_{h}\right)\right), P_{u}^{\perp}\left(\rho \boldsymbol{a}_{h} \cdot \nabla\left(\boldsymbol{u}-\boldsymbol{u}_{h}\right)\right)\right\rangle_{K} \\
& -\sum_{K} \alpha_{u}\left\langle P_{u}^{\perp}\left(\nabla \cdot\left(\boldsymbol{S}-\tilde{\boldsymbol{S}}_{h}\right)\right), P_{u}^{\perp}\left((1-\beta) \nabla \cdot\left(\boldsymbol{\sigma}-\boldsymbol{\sigma}_{h}\right)\right)\right\rangle_{K}
\end{aligned}
$$

The following bounds can be easily obtained using inverse estimates and the stability of the adjoint problem:

$$
\begin{aligned}
& \sum_{K} \alpha_{u}\left\langle P_{u}^{\perp}\left(\nabla\left(\pi-\tilde{\pi}_{h}\right)\right), P_{u}^{\perp}\left(\nabla\left(p-p_{h}\right)\right)\right\rangle_{K} \lesssim \sum_{K} \alpha_{u} \frac{\mu}{l^{2}}\left\|\boldsymbol{u}-\boldsymbol{u}_{h}\right\|_{K} \frac{1}{h}\left\|p-p_{h}\right\|_{K} \\
& \sum_{K} \alpha_{u}\left\langle P_{u}^{\perp}\left(\rho \boldsymbol{a}_{h} \cdot \nabla\left(\boldsymbol{w}-\tilde{\boldsymbol{w}}_{h}\right)\right), P_{u}^{\perp}\left(\rho \boldsymbol{a}_{h} \cdot \nabla\left(\boldsymbol{u}-\boldsymbol{u}_{h}\right)\right)\right\rangle_{K} \\
& \quad \lesssim \sum_{K} \alpha_{u}\left(\rho\left\|\boldsymbol{a}_{h}\right\|_{K}\right)^{2} \frac{h}{l^{2}}\left\|\boldsymbol{u}-\boldsymbol{u}_{h}\right\|_{K}\left\|\boldsymbol{u}-\boldsymbol{u}_{h}\right\|_{H^{1}(K)} \\
& \sum_{K} \alpha_{u}\left\langle P_{u}^{\perp}\left(\nabla \cdot\left(\boldsymbol{S}-\tilde{\boldsymbol{S}}_{h}\right)\right), P_{u}^{\perp}\left((1-\beta) \nabla \cdot\left(\boldsymbol{\sigma}-\boldsymbol{\sigma}_{h}\right)\right)\right\rangle_{K} \\
& \quad \lesssim(1-\beta) \sum_{K} \alpha_{u} \frac{\mu}{l^{2}}\left\|\boldsymbol{u}-\boldsymbol{u}_{h}\right\|_{K} \frac{1}{h}\left\|\boldsymbol{\sigma}-\boldsymbol{\sigma}_{h}\right\|_{K}
\end{aligned}
$$

Finally we need a bound for

$$
B_{\text {stab }}\left(\left[\boldsymbol{u}-\boldsymbol{u}_{h}, p-p_{h}, \boldsymbol{\sigma}-\boldsymbol{\sigma}_{h}\right],\left[\boldsymbol{w}-\tilde{\boldsymbol{w}}_{h}, \pi-\tilde{\pi}_{h}, \boldsymbol{S}-\tilde{\boldsymbol{S}}_{h}\right]\right)
$$


in (40). It can be bounded in terms of similar bounds as above, again using inverse and error estimates on the boundary, the stability of the adjoint problem and the expression of the stabilization parameters. We omit the details. When all these bounds are combined in (40) and finally used in (39) we obtain

$$
\begin{aligned}
& \frac{\mu}{l^{2}}\left\|\boldsymbol{u}-\boldsymbol{u}_{h}\right\|^{2} \lesssim h \frac{\sqrt{\mu}}{l^{2}}\left\|\boldsymbol{u}-\boldsymbol{u}_{h}\right\|\left(\sqrt{\mu}\left\|\boldsymbol{u}-\boldsymbol{u}_{h}\right\|_{H^{1}(\Omega)}+\sum_{K}\left(\frac{\rho\left\|\boldsymbol{a}_{h}\right\|_{K} h}{\mu}\right) \sqrt{\mu}\left\|\boldsymbol{u}-\boldsymbol{u}_{h}\right\|_{H^{1}(K)}+\frac{1}{\sqrt{\mu}}\left\|\boldsymbol{\sigma}-\boldsymbol{\sigma}_{h}\right\|\right. \\
& \left.+\frac{1}{\sqrt{\mu}} \sum_{K}\left(\frac{\lambda\left\|\boldsymbol{a}_{h}\right\|_{K}}{h}\right)\left\|\boldsymbol{\sigma}-\boldsymbol{\sigma}_{h}\right\|_{K}+\frac{1}{\sqrt{\mu}}\left\|p-p_{h}\right\|\right)
\end{aligned}
$$

and the theorem follows. Note that the bound obtained explodes with the cell Reynolds and the cell Weissenberg numbers.

\section{Conclusions}

In this paper we have presented the numerical analysis of a finite element approximation of the linearized viscoelastic flow problem. The formulation in the nonlinear case was presented in the stationary case in [13]. This analysis has confirmed what it was already known from the numerical experiments, namely, that the method provides stable solutions that converge to the exact solutions at an optimal rate of convergence. In particular, we have shown this using a mesh dependent norm especially tailored for the stabilized problem and also the norm of the space where the continuous problem is posed. What is relevant from the analysis presented is that it clearly displays how the estimates obtained deteriorate as the cell Reynolds number and the cell Weissenberg number increase. The former decreases when the mesh size is reduced, but the having a bound on the latter imposes the relaxation time to be very small, which is an indication of the existence of the so called high Weissenberg number problem.

\section{ACKNOWLEDGMENTS}

E. Castillo gratefully acknowledges the support received from CONICYT (Programa de Formación de Capital Humano Avanzado/Becas-Chile Folio 72120080), and from the Universidad de Santiago de Chile.

R. Codina gratefully acknowledges the support received from the Catalan Goverment through the ICREA Acadèmia Research Program.

\section{REFERENCES}

[1] F. P. T. Baaijens, M. A. Hulsen, and P. D. Anderson. The Use of Mixed Finite Element Methods for Viscoelastic Fluid Flow Analysis. John Wiley \& Sons, Ltd, 2004.

[2] J. Baranger and D. Sandri. Finite element approximation of viscoelastic fluid flow: Existence of approximate solutions and error bounds. Numerische Mathematik, 63(1):13-27, 1992.

[3] M. A. Behr, L. P. Franca, and T. E. Tezduyar. Stabilized finite element methods for the velocity-pressure-stress formulation of incompressible flows. Computer Methods in Applied Mechanics and Engineering, 104(1):31-48, 1993.

[4] R. B. Bird, R. C. Amstrong, and O. Hassager. Dynamics of Polymeric Liquids, vol.1: Fluid Mechanics. Wiley, New York, 2nd edition, 1987.

[5] R. B. Bird, R. C. Amstrong, and O. Hassager. Dynamics of Polymeric Liquids, vol.2: Kinetic Theory. Wiley, New York, 2nd edition, 1987. 
[6] A. C. Bogaerds, W. M. Verbeeten, G. W. Peters, and F. P. Baaijens. 3d viscoelastic analysis of a polymer solution in a complex flow. Computer Methods in Applied Mechanics and Engineering, 180(3-4):413-430, 1999.

[7] A. Bonito and E. Burman. A continuous interior penalty method for viscoelastic flows. SIAM Journal on Scientific Computing, 30(3):1156-1177, 2007.

[8] A. Bonito, P. Clément, and M. Picasso. Mathematical and numerical analysis of a simplified time-dependent viscoelastic flow. Numerische Mathematik, 107(2):213-255, 2007.

[9] J. Bonvin, M. Picasso, and R. Stenberg. GLS and EVSS methods for a three-field Stokes problem arising from viscoelastic flows. Computer Methods in Applied Mechanics and Engineering, 190(29-30):3893-3914, 2001.

[10] F. Brezzi and M. Fortin. Variational formulations and finite element methods. In F. Brezzi and M. Fortin, editors, Mixed and Hybrid Finite Element Methods, volume 15 of Springer Series in Computational Mathematics, pages 1-35. Springer New York, 1991.

[11] E. Castillo, J. Baiges, and R. Codina. Approximation of the two-fluid flow problem for viscoelastic fluids using the level set method and pressure enriched finite element shape functions. Journal of Non-Newtonian Fluid Mechanics, 225:37-53, 2015.

[12] E. Castillo and R. Codina. Stabilized stress-velocity-pressure finite element formulations of the Navier-Stokes problem for fluids with non-linear viscosity. Computer Methods in Applied Mechanics and Engineering, 279:554-578, 2014.

[13] E. Castillo and R. Codina. Variational multi-scale stabilized formulations for the stationary three-field incompressible viscoelastic flow problem. Computer Methods in Applied Mechanics and Engineering, 279:579-605, 2014.

[14] E. Castillo and R. Codina. First, second and third order fractional step methods for the three-field viscoelastic flow problem. Journal of Computational Physics, 296:113-137, 2015.

[15] R. P. Chhabra and J. F. Richardson. In Non-Newtonian Flow and Applied Rheology. Elsevier Butterworth-Heinemann, second edition, 2008.

[16] R. Codina. Stabilization of incompressibility and convection through orthogonal sub-scales in finite element methods. Computer Methods in Applied Mechanics and Engineering, 190(13-14):1579-1599, 2000.

[17] R. Codina. Stabilized finite element approximation of transient incompressible flows using orthogonal subscales. Computer Methods in Applied Mechanics and Engineering, 191(39-40):4295-4321, 2002.

[18] R. Codina. Finite element approximation of the three-field formulation of the Stokes problem using arbitrary interpolations. SIAM Journal on Numerical Analysis, 47(1):699-718, 2009.

[19] R. Codina and J. Blasco. A finite element formulation for the Stokes problem allowing equal velocity-pressure interpolation. Computer Methods in Applied Mechanics and Engineering, 143(3-4):373-391, 1997.

[20] R. Codina, J. Principe, and J. Baiges. Subscales on the element boundaries in the variational two-scale finite element method. Computer Methods in Applied Mechanics and Engineering, 198(5-8):838-852, 2009.

[21] V. J. Ervin, H. K. Lee, and L. N. Ntasin. Analysis of the Oseen-viscoelastic fluid flow problem. Journal of Non-Newtonian Fluid Mechanics, 127(2-3):157-168, 2005.

[22] V. J. Ervin and W. W. Miles. Approximation of time-dependent viscoelastic fluid flow: SUPG approximation. SIAM Journal on Numerical Analysis, 41(2):457-486, 2003.

[23] Y. Fan, R. Tanner, and N. Phan-Thien. Galerkin/least-square finite-element methods for steady viscoelastic flows. Journal of Non-Newtonian Fluid Mechanics, 84(2-3):233-256, 1999.

[24] R. Fattal and R. Kupferman. Constitutive laws for the matrix-logarithm of the conformation tensor. Journal of NonNewtonian Fluid Mechanics, 123(2-3):281-285, 2004.

[25] E. Fernández-Cara, F. Guillén, and R. Ortega. Mathematical modeling and analysis of viscoelastic fluids of the Oldroyd kind, in Handbook of Numerical Analysis, VIII. North-Holland, 2002.

[26] C. Guillopé and J. Saut. Existence results for the flow of viscoelastic fluids with a differential constitutive law. Nonlinear Analysis: Theory, Methods \& Applications, 15(9):849-869, 1990.

[27] J. S. Howell. Computation of viscoelastic fluid flows using continuation methods. Journal of Computational and Applied Mathematics, 225(1):187-201, 2009.

[28] T. J. Hughes, G. R. Feijóo, L. Mazzei, and J.-B. Quincy. The variational multiscale method-a paradigm for computational mechanics. Computer Methods in Applied Mechanics and Engineering, 166(1-2):3-24, 1998.

[29] M. A. Hulsen, R. Fattal, and R. Kupferman. Flow of viscoelastic fluids past a cylinder at high Weissenberg number: Stabilized simulations using matrix logarithms. Journal of Non-Newtonian Fluid Mechanics, 127:27-39, 2005. 
[30] P. Knobloch and L. Tobiska. On Korn's first inequality for quadrilateral nonconforming finite elements of first order approximation properties. International Journal of Numerical Analysis and Modelling, 2(4):439-458, 2005.

[31] J. Kwack and A. Masud. A three-field formulation for incompressible viscoelastic fluids. International Journal of Engineering Science, 48(11):1413-1432, 2010. Special Issue in Honor of K.R. Rajagopal.

[32] H. Lee. A multigrid method for viscoelastic fluid flow. SIAM Journal on Numerical Analysis, 42(1):109-129, 2004.

[33] P. L. Lions and N. Masmoudi. Global solutions for some Oldroyd models of non-Newtonian flows. Chinese Annals of Mathematics, 21(3):131-146, 2000.

[34] J. Marchal and M. Crochet. A new mixed finite element for calculating viscoelastic flow. Journal of Non-Newtonian Fluid Mechanics, 26(1):77-114, 1987.

[35] M. Picasso and J. Rappaz. Existence, a priori and a posteriori error estimates for a nonlinear three-field problem arising from Oldroyd-B viscoelastic flows. ESAIM: Mathematical Modelling and Numerical Analysis, 35(5):879-897, 2001.

[36] M. Renardy. Existence of slow steady flows of viscoelastic fluids with differential constitutive equations. ZAMM Journal of Applied Mathematics and Mechanics, 65(9):449-451, 1985.

[37] M. Renardy. Mathematical Analysis of Viscoelastic Flows. CBMS-NSF Regional Conference Series in Applied Mathematics, 1989.

[38] M. Renardy. Asymptotic structure of the stress field in flow past a cylinder at high Weissenberg number. Journal of Non-Newtonian Fluid Mechanics, 90(1):13-23, 2000.

[39] M. Renardy, W. Hrusa, and J. Nohel. In Mathematical Problems in Viscoelasticity. John Wiley \& Sons, Inc., New York, 1987.

[40] D. Sandri. Finite element approximation of viscoelastic fluid flow: existence of approximate solutions and error bounds. continuous approximation of the stress. SIAM Journal on Numerical Analysis, 31(2):362-377, 1994. 